\title{
How Fast Can You Trade? High Frequency Trading in Dynamic Limit Order Markets
}

\begin{abstract}
We consider a dynamic equilibrium model of high frequency trading (HFT). The model is a stochastic sequential game with endogenous trading decisions in a limit order market. There are two types of agents: fast and slow traders. Fast traders have speed advantages in terms of analysing information and the low-latency transmission of orders. Nevertheless, slow traders can observe and learn from the signals disclosed by fast traders in the market activity. We find that HFT improves market quality by increasing informational efficiency and liquidity. Fast traders make larger average trading profits than slow traders; however the trading profits of slow traders are higher when there is HFT in the market than in the case where HFT is absent. This is due to both the improvements in market quality induced by HFT and the learning process followed by slow traders, which increases the effectiveness of their trading strategies. We report that a cancellation fee, which has already been imposed by some exchanges, may affect negatively the market quality. Finally, we show that HFT traders may have incentives to manipulate market volatility since they can make larger profits through limit orders when we simulate a volatility shock.
\end{abstract}


Financial markets have undergone a major technological transformation during the past decade: from human-led transactions (at least pressing the "button") to technologies permitting high frequency trading, in which sophisticated computers quickly process information and automatically submit orders utilizing superfast connections to the exchanges. However, this financial innovation has generated a relatively favourable position for investors with high frequency trading (henceforth, HFT) technology, in terms of speed advantages over the rest of the market participants. High frequency traders have two main speed advantages: an informational advantage (fast access and quick analysis of market information); and a trading submission speed advantage (the low-latency transmission of orders and prompt modifications to previous trading decisions). Currently, there is a growing theoretical literature on understanding the impact of HFT on market quality and stability as well as possible damage to 'traditional' investors. These studies have mainly characterized HFT through the first speed feature described above: the informational advantage. ${ }^{1}$ Nevertheless, there has been a limited number of efforts in the financial economics literature aimed at developing a model in which high frequency traders have an effective superior trading submission speed. The main goal of our study is to fill this gap by presenting a dynamic equilibrium model with HFT in a limit order market, in which we incorporate all the speed characteristics of HFT technology in an environment with diverse types of traders.

The trading speed advantage, reflected in the low-latency transmission of orders and prompt modifications to previous trading decisions, is a key feature of HFT technology. For instance, suppose all market participants have the same level of information (i.e. there is no informational advantage), but there is a group of HFT traders who can submit orders with a lower latency and can revise and modify previous strategies faster than 'traditional' slow traders. On the one hand, HFT traders can submit quick orders to obtain the major part of the benefits of any possible difference between the asset price and its fundamental value. On the other hand, in

\footnotetext{
${ }^{1}$ See, e.g. Martinez and Roşu (2011), Biais et al. (2012a), and Foucault et al. (2012).
} 
the case that a slow trader makes a trading decision today, she faces adverse selection if the market condition unfavourably changes in the future, because she cannot react and modify her previous trading strategy as quickly as the fast traders. In this scenario, fast traders can submit quick orders against this slow trader to increase their trading profits even more. In fact, the trading speed advantage has been recognized by academics and regulators. For instance, Hasbrouck and Saar (2012) write: "We define low-latency activity as strategies that respond to market events in the millisecond environment, the hallmark of proprietary trading by high-frequency trading firms". In addition, in 2010 the SEC stated that: "characteristics often attributed to proprietary firms engaged in HFT are: the use of extraordinarily high speed and sophisticated computer programs for generating, routing, and executing orders".

We present a dynamic equilibrium model in continuous-time with a single asset. The model is a stochastic asynchronous game with endogenous trading decisions in a limit order market. The common value of the asset, $v_{t}$, follows a random walk and reflects its fundamental valuation. ${ }^{2}$ There are two types of risk-neutral traders: fast traders and slow traders (also called HFT traders and 'traditional' traders, respectively). Fast traders have superior speed in terms of: analysing information; and trading submission which is reflected in the low-latency transmission of orders together with quick revisions and prompt modifications to previous trading decisions. First, fast traders can contemporaneously observe $v_{t}$, while slow traders observe the fundamental value of the asset with a time lag (i.e. at any instant $t$ slow traders only know $\left.v_{t-\Delta_{t}}\right){ }^{3}$ Second, traders arrive at the market sequentially and randomly following two Poisson processes with parameters $\lambda^{H F T}$ for fast traders and $\lambda^{S T}$ for slow traders, where $\lambda^{H F T}>\lambda^{S T}{ }^{4}$ In addition, traders can re-enter at the market multiple times to revise and to modify previous trading strategies. However, agents

\footnotetext{
2 The fundamental value of the asset can be thought of as the discounted value of expected future dividends.

3 This assumption is supported by previous empirical studies on HFT, which show that fast traders are better informed than other market participants (see, e.g., Hendershott and Riordan, 2010; Brogaard, 2010; Kirilenko et al., 2011; and Brogaard et al., 2011). In addition, similar assumptions have already been used in HFT theoretical models by Biais et al. (2012a), Foucault et al. (2012), and Martinez and Roșu (2011).

${ }^{4}$ The expected time between arrivals for high frequency traders is lower than for slow traders, since the expected value of an exponentially distributed variable $x, E(x)$, with parameter $\lambda$ is $E(x)=1 / \lambda$.
} 
cannot instantaneously modify trading decisions due to the fact that cognition limits prevent them from continuously monitoring the market; thus trading plans are 'sticky' (see, e.g., Biais et al., 2012b). Nevertheless, high frequency traders have the possibility of evaluating market changes and modifying previous trading strategies much faster than slow traders. Thus, fast traders and slow traders re-enter at the market according to two Poisson processes at rate $\lambda_{r}^{H F T}$ and $\lambda_{r}^{S T}$, respectively, where $\lambda_{r}^{H F T}>\lambda_{r}^{S T}$.

Currently, the exchanges in which we can find HFT are fully, or at least partially, organized as limit order markets (e.g. BATS U.S. stock exchange, NYSE, NASDAQ, London Stock Exchange, NYSE Euronext, BATS Chi-X Europe). ${ }^{5}$ Consequently, the microstructure features and particularities of these types of trading venues should be considered when evaluating the effects of HFT on market quality and stability. ${ }^{6}$ Therefore, we consider a limit order market in our dynamic equilibrium model. Traders can submit market orders or limit orders. ${ }^{7}$ As in a real limit order market, the limit order book is characterized by a set of discrete prices, and respects the time and price priorities for the execution of limit orders. Consequently, given the dynamic features of our equilibrium model, we can generate a complete limit order book over time, which represents an additional contribution by our paper. In fact, a recent study of HFT technology sponsored by the British government states that: "simulation tools and techniques could enable central regulatory authorities to judge the stability of particular financial markets, given knowledge of the structure of those markets". ${ }^{8}$ In our study, we simulate a historical limit order book; thus we are able to simultaneously evaluate the impact of HFT from multiple edges and scenarios, to analyse the dynamic

\footnotetext{
${ }^{5}$ In fact, $85 \%$ of the leading stock exchanges around the world are now entirely electronic limit order markets with no floor-trading (Jain, 2005).

${ }^{6}$ HFT traders have to take into account the microstructure characteristics of markets when they design their investment strategies, which also makes features of limit order markets relevant to evaluate the impact of HFT on market quality and stability.

${ }^{7} \mathrm{~A}$ limit order is a commitment made by a trader at time $t$ to trade the asset in the future at a prespecified price $p$; while a market order is a request to trade right now at the best price available (i.e. at the bid or ask prices depending the direction of the order). In addition, a limit order from a given trader is always executed through the submission of a market order.

8 This study involved 150 leading experts from more than 20 countries. The name of the study is "Foresight: The Future of Computer Trading in Financial Markets (2012) Final Project Report".
} 
interactions between different types of traders, and to examine several market quality measures.

We find that HFT reduces microstructure noise since it mitigates the cognitive limits of human beings. In addition, despite the fact that slow traders can observe the fundamental value of the asset with a time lag $\Delta_{t}$, in the model we allow them to capture and to learn from the information revealed in the market activity by HFT traders. The learning process followed by slow traders helps them to make more precise estimations about the contemporaneous fundamental value of the asset, and thus to make better trading decisions. Consequently, the cognitive capacity of slow traders combined with the presence of HFT traders in the market induce a reduction in the slow trader's errors in beliefs in relation to $v_{t}$. Our findings are consistent with the empirical evidence reported in Hendershott and Riordan (2010), Brogaard (2010), and Brogaard et al. (2012) regarding the improvements in informational efficiency generated by HFT in markets. ${ }^{9}$

Slow traders prefer to submit more market orders than fast traders since limit orders have the risk of being 'picked-off' when market conditions change unfavorably. The 'picking-off risk' of limit orders is particularly high for 'traditional' slow agents when HFT traders are present, because HFT generates informational and submission speed advantages to agents with this technology. HFT traders prefer to submit limit orders, and thus they are mainly liquidity suppliers (see Jovanovic and Menkveld, 2011). Even though the reasons for the submission habits of traders are both informational differences and trading speed differences, we show that the latter has a larger impact on trading behaviour.

HFT improves the depth of the limit order book since fast traders prefer to submit more limit orders, which also results in more informative and competitive market quotes. Thus, the bid-ask spread decreases when there is HFT. The bid-ask spread reduction is also attributable to the high market liquidity since there are more chances to find a counterpart for an order submission, and due to the improvement in

\footnotetext{
${ }_{9}$ Our findings are also related to Kirilenko et al. (2011), who provide empirical evidence that high frequency traders may have informational advantages, since they can make orders in the right direction in relation to price changes.
} 
market informational efficiency described previously. ${ }^{10}$ Our results are also congruent with the results of empirical studies which show that there is a positive relation between HFT and market liquidity (see, e.g., Hendershott et al., 2012; Hasbrouck and Saar, 2012; and Riordan and Storkenmaier, 2012).

HFT traders make larger trading profits than slow traders. However, the trading profits of slow traders are higher when there is HFT in the market than in the case where HFT is absent. This is due to the improvement in market quality generated by HFT, which produces an 'indirect' economic gain for slow traders. When we independently analyse the effect of the HFT advantages on the economic differences between slow and fast traders, the trading speed advantage of HFT traders has a larger impact. This is due to the cognitive capacity of slow traders, who can learn from the knowledge disclosed by HFT traders in the order submissions, thereby reducing the informational differences between agents.

The model is a sequential trading game in which traders have to make several rational trading decisions endogenously. Trading decisions include the submission of an order or the decision to await subsequent periods in the game, to submit a buy or a sell order, the choice between market and limit orders, and the submission price in the case of a limit order. Additionally, traders can re-enter at the market to modify their unexecuted limit orders. The re-entering process implies additional trading decisions on whether to cancel a previous limit order but face a cancellation cost, or to wait until the order is executed in subsequent periods, which involve a cumulative cost for not executing the order immediately. In the case of a cancellation, traders can submit a new order or wait for different market conditions in the future for a potential order submission. Traders who submit limit orders remain part of the trading game by revising their unexecuted orders; but they exit the market permanently once their orders have been executed. We incorporate additional heterogeneity for the agents, since they have diverse private values in addition to the common value of the asset. The private value represents intrinsic trading motives, such as tax exposures, wealth shocks, hedging needs or differences in investment horizons, amongst others.

\footnotetext{
10 See Copeland and Galai (1983), Glosten and Milgrom (1985), and Kyle (1985) for an explanation of the relationship between the bid-ask spread and market informational efficiency.
} 
Market conditions (e.g. shape of the limit order book and the fundamental value of the asset) affect the expected utility of traders when they submit an order. Therefore, market conditions endogenously affect the decision process followed by the agents, and thus optimal trading decisions are state-dependent. Moreover, traders have to resolve a dynamic maximization problem. On the one hand, traders have to take into account the fact that their current actions will affect the future strategies of other agents, and thus future market conditions and prospective payoffs. On the other hand, traders have to consider the possibility of future re-entries, including potential cancellations and resubmissions, which imply further dynamic characteristics to the model.

Agents maximize their utility that is represented in the Bellman equation for the dynamic decision problem, which allows us to calculate the welfare gains for different traders' decisions. We obtain the equilibrium numerically, as the model is analytically intractable. Given the asynchronous nature of the game, we solve the equilibrium using the algorithm introduced by Pakes and McGuire (2001), which was originally proposed for industrial organization problems with sequential decisions. This algorithm provides a Markov-perfect equilibrium which has been successfully implanted into a dynamic model for limit order markets by Goettler et al. (2005, 2009), although without exploring the effects of HFT on market quality and stability as in our study. Once we obtain the model equilibrium, we simulate time series of orders and trades, and the complete evolution of the order book as in an authentic limit order market.

Finally, we perform two policy exercises through our model. We examine the impact of different cancellation fees and different volatility shocks on the market quality and stability. First, the intended effect of a cancellation fee, which has already been imposed by some exchanges, is to prevent bad practices in HFT such as 'quote stuffing'.11 We find that a cancellation fee reduces the number of cancellations by both HFT and non-HFT traders, but it also negatively affects market quality. Second, the

\footnotetext{
11 'Quote stuffing' is the tactic of rapidly submitting and cancelling a large quantity of limit orders, to generate an avalanche of useless information that other agents have to analyze, with the objective of diminishing the possible competitiveness of other agents.
} 
analysis of a volatility shock helps us to understand whether HFT may affect market stability. This analysis shows that HFT traders may have an incentive to manipulate market volatility since they can make larger profits through limit orders when there is a volatility shock. This incentive may affect market stability because a limit order market is a 'volatility multiplier'. ${ }^{12}$ This finding is also consistent with the study of Kirilenko et al. (2011) in relation to the 'flash crash'. Kirilenko et al. (2011) find that the 'flash crash' was due to a wrongly executed selling plan by a large fundamental trader; nevertheless the abnormal trading behaviours by high frequency traders exacerbated the unusual market volatility.

The study of the effects of HFT on market quality and stability is extremely relevant given the large proportion of trading activity that is generated by HFT traders. ${ }^{13}$ To the best of our knowledge, no theoretical studies of HFT exist in which the main speed advantages of this technology are simultaneously studied. In addition, we present a dynamic equilibrium model that includes the microstructure features of a true limit order book, which allows the analysis of diverse market quality measures and the evaluation of potential policy regulations as in a real market. Therefore, as previously stated, the focus of our study on the impact of HFT on dynamic limit order markets by evaluating the main characteristics of this technology appears distinctive. The paper is organised as follows. Section 2 presents a literature review. Section 3 introduces the model. Section 4 describes the algorithm used to solve the asynchronous trading game and the model parameterization. Section 5 shows the effects of HFT on microstructure noise and errors in slow traders' beliefs. Section 6 presents the relationship between HFT and the submission behaviour of market participants. Section 7 analyses the impact of HFT on the bid-ask spread and the limit order book depth. Section 8 examines the effects of HFT on average payoffs for traders. Section 9 reports two policy analyses (the effect of different cancellation fees and the impact of diverse volatility shocks on market quality and stability). Finally, section 10 concludes.

\footnotetext{
12 Goettler et al. (2009) also show that a limit order market is a 'volatility multiplier'.

${ }^{13}$ For instance, $73 \%$ of the trading volume on the U.S. stock market in 2009 can be attributed to highfrequency proprietary trading; as compared to practically zero in the mid-1990s (see Hendershott et al., 2011). Similar results have been obtained in empirical studies for NASDAQ (Brogaard, 2010) and for foreign exchange markets (Chaboud et al., 2011).
} 
Our work is connected to the growing theoretical literature on HFT. However, previous studies have not explored the important features of HFT technology in relation to the low-latency transmission of orders and the quick revisions and modifications to previous trading strategies. In addition, they do not fully include the important and essential relationship between HFT and the microstructure features of dynamic limit order markets, as our study does. Biais et al. (2012a) present a 3-period model of HFT, in which fast traders know the fundamental value before slow traders in a way which is similar to our approach. Biais et al. (2012a) find that fast traders can generate adverse selection costs for slow traders; and thus HFT may induce negative externalities. They argue that adverse selection appears due to the superior information of fast traders, given that high frequency traders can process public information faster than slow agents. Foucault et al. (2012) present two dynamic models with a market maker and an informed trader who can only submit market orders. In the first model, the market maker and the trader receive information at the same time (although with different precision levels); while in the second model the informed trader receives information a moment before the market maker. Foucault et al. (2012) find that the speed advantage in information increases trading volume, decreases liquidity, induces price changes that are more correlated with fundamental value movements, and reduces informed order flow autocorrelations.

Martinez and Roşu (2011) introduce a model with a dealer and informed fast traders. HFT traders only submit market orders and have an informational advantage, but they are also uncertainty averse regarding the level of the asset value. Martinez and Roşu (2011) find that HFT generates most of the volatility and trading volume in the market, and present evidence that HFT makes the markets more efficient as fast traders incorporate their information advantages in transaction prices. ${ }^{14}$ Jovanovic and Menkveld (2011) present a model of HFT liquidity suppliers with access to public information. Jovanovic and Menkveld (2011) show that fast liquidity suppliers may

\footnotetext{
${ }^{14}$ Additionally, Pagnotta and Philippon (2012) study exchanges' incentives to invest in faster platforms. They show that exchange competition in speed reduces prices further, leads to more fragmentation, improves investor participation and increases the trading volume.
} 
reduce informational friction due to the superior speed in information analysis and execution of HFT technology, but that HFT can also reduce welfare because of the adverse selection that slow traders face.

Our study is methodologically associated with the state-of-the-art microstructure models for limit order markets developed by Goettler et al. $(2005,2009)$. Goettler et al. $(2005,2009)$ introduce dynamic models in which investors have to make asynchronous trading decisions, depending on their information set and the market structure, in which the equilibrium is obtained numerically as in our study. Their model represents a step forward in terms of realism in relation to previous multiperiod models of limit order markets. ${ }^{15}$ Even though there is a methodological connection between our paper and the microstructure study conducted by Goettler et al. $(2005,2009)$, our research focus differs in exploring the impacts of HFT in relation to market quality and integrity; and thus our objective is to answer a different set of questions for the financial economics literature. Furthermore, we consider a more developed model for HFT that includes traders with different speeds in the lowlatency transmission of orders, and different speeds in the revision of and modifications to previous trading strategies. Additionally, we perform policy analyses by including a cancellation fee in the model to avoid anticompetitive tactics by high frequency traders (which has already been implemented in some markets); and we evaluate the effects of different volatility shocks on market quality and stability. ${ }^{16}$

\footnotetext{
15 Early work on multi-period equilibrium models for limit order markets imposed some restrictive assumptions to make the models analytically tractable (see, e.g., Parlour, 1998; Foucault, 1999; Foucault et al., 2005; and Roşu, 2009).

16 Our paper is closely related to Biais et al. (2012b), who present a model in which investors have sticky plans due to limited cognition. Although Biais et al. (2012b) do not specifically study the interaction between fast and slow traders and the possible informational advantages of HFT technology, they analyze the effects of sticky trading decisions in a limit order market. They show that sticky trading plans lengthen market price recovery and induce round trip trades which increase volume. See Lynch (1996), Reis (2006a,b), Mankiw and Reis (2002), Alvarez et al. (2011), and Alvarez et al. (2012) for additional studies regarding the economic impact of infrequent updating in investment decisions.
} 


\section{$3 \quad$ The model}

\subsection{The market characteristics}

We consider a dynamic continuous-time model of high frequency trading in a limit order market with a single financial asset. The fundamental value of the asset, $v_{t}$, follows a random walk with drift zero and volatility $\sigma$. The model is an asynchronous dynamic trading game in which there are two types of risk-neutral traders: fast traders and slow traders. HFT traders can process new information faster than slow traders. Similar to Biais et al. (2012a), Foucault et al. (2012) and Martinez and Roşu (2011), we assume that at time $t$ fast traders know the current fundamental value of the asset $v_{t}$; while slow traders only know the fundamental value with a lag $\Delta_{t}$. Fast traders arrive at the market following a Poisson process at rate $\lambda^{H F T}$, while slow traders also arrive according to a Poisson process at rate $\lambda^{S T}$, where $\lambda^{H F T}>\lambda^{S T}$. Traders can submit limit orders and market orders. Traders can revise and modify their unexecuted limit orders multiple times. However, due to cognition limits, traders cannot immediately modify their previous limit orders after a change in the market conditions; thus trading decisions are 'sticky'. Nevertheless, HFT traders have more tools and resources to evaluate possible cancellations and thus they can make modifications faster than slow agents. Fast traders re-enter at the market following a Poisson process at rate $\lambda_{r}^{H F T}$, while slow traders also re-enter according to a Poisson process at rate $\lambda_{r}^{S T}$, where $\lambda_{r}^{H F T}>\lambda_{r}^{S T}{ }^{17}$

All traders observe the evolution of the order book until time $t$, which generates two informational effects. On one hand, slow traders can use the historical trading activity to update their expectations of the fundamental value of the asset, and hence to make a better prediction of the current value of $v_{t}$. On the other hand, high frequency traders also observe the trading history of the market and can also estimate the expected value of slow traders regarding $v_{t}$; and thus fast agents can predict the trading strategies of slow traders, which enables them to further increase the payoffs related to HFT technology.

\footnotetext{
17 We use a similar notation to Goettler et al. (2005 and 2009) regarding the microstructure features of the model for the dynamic order book market.
} 
Each trader has a private value $\alpha$ for the asset, which is drawn from a distribution $F_{\alpha}$ and known before making any trading decision. The private value is idiosyncratic and constant to each agent. The private value arises from differences in terms of intrinsic benefits from trading such as tax exposures, wealth shocks, hedging needs, or differences in investment horizons, amongst others. This private value gives additional heterogeneity to the different agents in the dynamic trading game. For instance, traders with $\alpha$ equal to zero (and hence with no intrinsic benefits to trade) are indifferent in taking either side of the market and hence maximize their benefits depending on the available trading possibilities; consequently they are likely liquidity suppliers since they will probably submit limit orders. Conversely, traders with higher absolute values in their intrinsic benefits to trade are likely to be liquidity demanders. ${ }^{18}$

As in real limit order markets, the limit order book $L_{t}$ is described by a discrete set of prices, denoted as $\left\{p^{i}\right\}_{i=-\infty}^{\infty}$, where the tick size, $d$, is the distance between any two consecutive prices. There is a backlog of outstanding orders to buy or to sell, $l_{t}^{i}$, which are associated with each price $p^{i}$. A positive (negative) number in $l_{t}^{i}$ denotes buy (sell) orders in the book, and it represents the depth at price $p^{i}$. Therefore, the bid price is $B\left(L_{t}\right)=\max \left\{p^{i} \mid l_{t}^{i}>0\right\}$ while the ask price is $A\left(L_{t}\right)=\min \left\{p^{i} \mid l_{t}^{i}<0\right\}$, and if the order book is empty on the bid side or on the ask side $B\left(L_{t}\right)=-\infty$ or $A\left(L_{t}\right)=\infty$, respectively.

The limit order book respects the time and price priorities for the execution of limit orders. Buy (sell) orders at higher (lower) prices are executed first, and limit orders submitted earlier have priority in the queue when they have the same price. In addition, when a trader submits an order, the order price identifies whether the order is a market order or a limit order. This means that an order to buy (sell) at a price above (below) the ask (bid) price is executed immediately at the ask (bid) price; and thus this order is a market order.

${ }^{18}$ Fast traders with zero private value are equivalent to the HFT liquidity suppliers in Jovanovic and Menkveld (2011). 
Each agent can trade one share and has to make three main trading decisions after arriving in the market: i) to submit an order or to wait until the market conditions change; ii) to buy or to sell the asset; and iii) to choose the price at which she will submit the order, which implies the decision to submit a market order or a limit order, depending on whether the price is above or below the quotes. ${ }^{19}$ Therefore, despite the fact that traders arrive following the Poisson processes with parameters $\lambda^{H F}$ and $\lambda^{S F}$, the submission rate is different as agents can decide to submit or to wait in the market, which depends endogenously on the market conditions given that all trading decisions are state-dependent.

As we mentioned previously, traders can re-enter the market and modify their previous unexecuted limit orders. Therefore, traders have to make additional trading decisions after re-entering at the market: i) whether to cancel an unexecuted limit order or retaining the order without changes; ii) if she decides to cancel the order, whether to submit a new order (a limit or a market order) for the asset or wait for different market conditions in the future to submit an order; and iii) if she decides to submit a new order after a cancellation, she has to choose the type of order and its price. Therefore, agents have to take the possibility of re-entry into account in the utility maximization problem.

Once a trader submits a limit order, she remains part of the trading game by revising her order until it is executed; however, after execution of the order the traders exit the market permanently. Consequently, there are a random number of active market participants at each instant, who are monitoring their previous limit orders.

Traders have to pay a cancellation fee $c_{\text {canc }}$ when they cancel an unexecuted submitted limit order. ${ }^{20}$ In the case of a re-entry, a trader can leave the order without changes, which has the benefit of keeping her priority time in the queue and avoiding

\footnotetext{
19 We can include additional shares per agent in the trading decision. However, similarly to Goettler et al. (2009), we assume one share per trader to make the model computationally tractable.

${ }^{20}$ We also obtain the model equilibrium and simulate a historical limit order book with a cancellation fee equal to zero (see Section 9). However, we include a cancellation cost in the model with the objective of evaluating recent regulations on some exchanges (e.g. NYSE Euronext), where there is a fee for cancellations to try to prevent uncompetitive practices in HFT such as 'quote stuffing'.
} 
a cancellation fee. ${ }^{21}$ The negative side of leaving an order in the book is that the asset value could move in directions that affect future payoffs. For instance, in the scenario of a growth in the asset value, some limit sell orders could be priced too low, and a quick trader could make profits from the difference. This possibility represents an implicit transaction cost of being 'picked off' when prices change unexpectedly after limit orders have been submitted. Conversely, when the asset value decreases, a sell limit order has the risk of not resulting in a trade. To take into account the risk that a limit order may not result in a trade, we include a cost of 'delaying' by a discount rate $\rho_{d}$, which reflects the cost of not executing immediately. This 'delaying' cost does not represent the time value of money; instead $\rho_{d}$ reflects opportunity costs and the cost of monitoring the market until a limit order is executed. Thus, the payoffs of order executions are discounted back to the order submission time at rate $\rho_{d}$.

\subsection{The trader's dynamic maximization problem}

Let $Y \in\{0.1\}$ be a trader indicator, where $Y=0$ if an agent is an HFT trader and $Y=1$ if the agent is a slow trader. Suppose that a trader arrives at the trading game and observes state $s$ of the market. For convenience, let the entry time be equal to zero. The state that a given trader observes includes: i) her private value $\alpha$; ii) her trading speed features $Y$; iii) the contemporaneous limit order book $L_{t}$ that results from previous trading activity, and the features of the previous transaction (price and whether it was due to a sell order or a buy order); iv) her current beliefs concerning the fundamental value of the asset, $v_{0}^{Y}$, that depends on $Y$; and $v$ ) the status of her previous action in the case that the trader has already submitted an earlier limit order to the market, which includes the original submission price, the priority in the book given that price, and the type of order. Recall that in the case of a fast trader, $v_{0}^{Y}$ is equal to the contemporaneous fundamental value of the asset (i.e. $v_{0}^{0}=v_{0}$ ). In the case of a slow trader, she can only know the fundamental value of the asset with a lag $\Delta_{t}$, but she can also observe previous trading activity up to the present, which allows

\footnotetext{
${ }^{21}$ It is important to point out that the order priority could have changed, depending on the shape of the
} book, which should be taken into account in the decision to cancel and re-submit. 
her to capture and to learn from the information disclosed by fast traders and thus to improve her accuracy concerning $v_{0}^{1}$ in relation to $v_{0}$.

Let $\Gamma(s)$ be the possible set of actions that a trader can take given the state $s$ (e.g. to wait until market conditions change, to buy or to sell the asset, and the submission prices, amongst others). Let $\eta(h \mid \tilde{a}, s)$ be the probability that an order is executed at time $h$ given that the trader takes the action $\tilde{a} \in \Gamma(s)$ when she faces the state $s$. It is important to notice that $\eta(\cdot)$ incorporates all possible future states and strategic actions adopted by other traders until $h$. If the decision $\tilde{a}$ is the submission of a market order $\eta(0 \mid \tilde{a}, s)=1$, while $\eta(h \mid \tilde{a}, s)$ converges asymptotically to zero when the trader decides to submit a limit order with a price far away from the fundamental value. In addition, let $\gamma(v \mid \sigma, s)$ be the density function of $v$ at time $h$ that depends on the volatility $\sigma$ of the fundamental value of the asset and the state $s$. The density function $\gamma(\cdot)$ depends on $s$ because it takes into account the current belief regarding the fundamental value of the asset, which is particularly important in the case of slow traders. Therefore, the expected value of an order that is executed prior to a re-entry at time $h_{r}$ is:

$$
\pi\left(h_{r}, \tilde{a}, s\right)=\int_{0}^{h_{r}} \int_{-\infty}^{\infty} e^{-\rho_{d} h_{r}}((\alpha+v-\tilde{p}) \tilde{x}) \eta\left(h_{r} \mid \tilde{a}, s\right) \gamma(v \mid \sigma, s) d v d h_{r}
$$

Here, $(\alpha+v-\tilde{p}) \tilde{x}$ is the instantaneous payoff of the order where $\tilde{p}$ is the submission price which is part of the decision $\tilde{a}$; while $\tilde{x}$ is also a component of the decision $\tilde{a}$ and reflects whether the trader decides to submit a buy order $(\tilde{x}=1)$, to submit a sell $\operatorname{order}(\tilde{x}=-1)$ or to submit nothing $(\tilde{x}=0)$. This payoff is transformed to a present value at the rate $\rho_{d}$ which is the cost of 'delaying' previously defined in this section.

Let $R(\cdot)$ be the probability distribution of the re-entry time which is exogenous and follows an exponential distribution at rates $\lambda_{r}^{H F T}$ or $\lambda_{r}^{S T}$ if the agent is a fast trader or a slow trader, respectively. In addition, let $\psi\left(s_{h_{r}} \mid \tilde{a}, s, h_{r}\right)$ be the probability that the state $s_{h_{r}}$ takes place at time $h_{r}$ given the previous state $s$ and the action $\tilde{a}$, which also includes all potential states and strategic decisions followed by other traders until $h_{r}$. 
Therefore, the value to an agent of arriving at the state $s, V(s)$, is given by the Bellman equation of the trader's optimization problem:

$$
\begin{aligned}
& V(s)=\max _{\tilde{a} \in \Gamma(s)} \int_{0}^{\infty}\left[\pi\left(h_{r}, \tilde{a}, s\right)+e^{-\rho_{d} h_{r}} \int_{s_{h_{r}} \in S}\left(V\left(s_{h_{r}}\right)-\tilde{z}_{s_{h_{r}}} c_{\text {canc }}\right)\right. \\
& \left.\cdot \psi\left(s_{h_{r}} \mid \tilde{a}, s, h_{r}\right) d s_{h_{r}}\right] d R\left(h_{r} \mid s\right)
\end{aligned}
$$

where $\tilde{z}_{S_{h_{r}}}=1$ if the optimal decision in the state $s_{h_{r}}$ is a cancellation and $\tilde{z}_{S_{h_{r}}}=0$ in any other case, and $S$ is the set of possible states. The first term is defined in equation (1); while the second term reflects the subsequent payoff in the case of re-entries.

\section{The algorithm used to solve the asynchronous trading game and the model parameterization}

We solve the model using a numerical method due to the analytical intractability of the trading game. Nevertheless, a solution using traditional numerical approaches is also difficult to obtain, given the large dimension of the state space of the model. For that reason, following Goettler et al. $(2005,2009)$, we obtain a stationary Markovperfect equilibrium using the algorithm introduced by Pakes and McGuire (2001), which resolves a large state space size problem by reaching the equilibrium only on the recurring states class. In this section, we will explain the algorithms used to reach the equilibrium, and later we will describe the plausible parameters used in our model that incorporate the relevant market features of limit order markets with the presence of 'traditional' slow traders and HFT traders.

The model reflects a dynamic trading game in which traders asynchronously arrive and select optimal actions (i.e. trading decisions) that maximize their expected utility given the observed state. Therefore, optimal trading decisions are state-dependent. Moreover, trading decisions are Markovian, since the market condition reflected in the observed state is a consequence of the history of events and previous states that define the game. 
The intuition behind the Pakes and McGuire's (2001) algorithm is that we can initially see the trading game as a Bayesian learning process in which traders learn how to behave in each state. Thus, traders follow a learning-by-doing process by playing in the game until we reach the equilibrium. In this learning-by-doing process, the trading game starts with each type of trader having initial beliefs about the expected payoffs of different actions and states. Afterwards, traders update their beliefs dynamically by playing in the game when they observe their realized payoffs from their actions. The equilibrium is reached when the expected payoff and the optimal trading decision, of each trader type in a given state $s^{*}$, are exactly the same expected payoff and decision if a similar trader observes $s^{*}$ in the future (i.e. there is nothing to learn anymore). Therefore, we obtain a Markov-perfect Bayesian equilibrium; which is also a symmetric equilibrium since it is time independent, because optimal trading decisions from each type of trader are the same when they face the same state in the present or in the future.

Once we obtain the equilibrium after making traders play in the game for a couple of billion trading events, we fix the traders' beliefs and simulate a further 300 million events. These last 300 million simulated events allow us to evaluate the behaviour of the different agents without the effects of learning process described in the previous paragraph. Consequently, all the results and analysis presented in this paper are obtained from the last 300 million simulated events. Despite the fact that the study of learning in an environment with HFT could be interesting to analyse, we prefer to leave this analysis for future research. Our objective in this paper is to evaluate the effects of HFT on market quality and integrity without capturing additional noise due to cognitive mechanisms.

HFT influences market quality in a highly nonlinear way given the properties of limit order market. Limit order markets have nonlinear features that make a simulation analysis necessary to understand the wide scope of outcomes that HFT induces in market performance. In this context, Kleidon (1986) shows that the use of standard tests to evaluate an equilibrium model using a single economy represented by market data could lead to inaccurate analysis. Kleidon (1986) points out that asset prices in equilibrium are calculated based on agents' expectations of future events across 
multiple and different economies. Therefore, the use of a specific realization given by market prices could induce wrong conclusions. Instead, Kleidon (1986) also proposes the use of multiple realizations by simulation techniques. Moreover, the use of simulation allows us to modify parameter setups and thus to observe the impacts of HFT on multiple environments.

We also fix the speed condition for each type of trader to solve the equilibrium of the model. Therefore, the cost of being 'fast' (i.e. the cost of having HFT technology) is given by the differences in payoffs for fast traders and slow traders. In the Appendix, we explain in detail the algorithm and convergence criteria used to obtain the model equilibrium. In addition, we use some features of the model to reduce the dimensionality of the state space, and we impose some specific restrictions with the objective of making the problem computationally tractable, which are also explained in detail in the Appendix.

We assume the following plausible parameter values to be used in the simulations of our base case. In the random walk process of the fundamental value of the asset we use a volatility, $\sigma$, equal to 0.50 on an annual basis. The value of the volatility is based on the analysis of Zhang (2010), who presents a daily volatility for U.S. stock returns of 0.033 , which is equivalent to 0.524 on an annual basis. In unreported results, we modify the value of $\sigma$ to evaluate the impact of the volatility on the agents' behaviour and market quality measures. We observe that the results are robust and comparable to the findings presented here when there are other volatility levels.

Similar to Goettler et al. (2009), we assume that the distribution of the private value is assumed to be discrete with support $\{-8,-4,0,4,8\}$ in ticks and with a cumulative distribution function $\{0.15,0.35,0.65,0.85,1.00\}$, which is based on the findings of Hollifield et al. (2006) regarding the private values of stocks on the Vancouver Stock Exchange.

We assume that slow traders arrive on average every 400 milliseconds (i.e. $\lambda^{S T}=1 / 0.40$ ). This is consistent with the literature in human behaviour in relation to reaction times. Reaction times of human beings are in the order of 200 milliseconds 
for a single stimulus to 700 milliseconds for six stimuli (see Kosinski, 2012). In addition, we arbitrarily assume that HFT traders are 20 times faster than slow traders; thus their average arrival time is 20 milliseconds (i.e. $\lambda^{H F T}=1 / 0.02$ ). This rate of arrivals for fast traders is also consistent with the timescale that Cont (2011) uses to define HFT traders, which is between one millisecond and 100 milliseconds. The main idea of this arrival rate for fast traders is to capture the impact on market quality and stability of differences in trading speed between slow and HFT traders. Moreover, limited cognition, due to the fact that traders are engaged in other tasks or because there is a noisy environment, may affect the modification speed of previous trading decisions. For instance, Trimmel and Poelzl (2006) show that background noise lengthened reaction time by inhibiting parts of the cerebral cortex, which may increase cognitive limits when multiple stimuli are received. In the model, we assume that high frequency traders re-enter the market to revise previous submitted orders on average every 80 milliseconds $\left(\lambda_{r}^{H F T}=1 / 0.08\right)$, which reflects the fact that even fast traders cannot monitor the market continuously because: there are computational processing times, there is noise in the signals received, and it is costly. However, HFT traders have more tools and resources for evaluating and monitoring their orders in the book than slow traders, and thus HFT traders are 20 times faster to re-enter the market than slow traders (i.e. $\lambda_{r}^{S T}=1 / 1.60$ ). We modify the relative speed between fast and slow traders in the analysis presented in the following sections, and the results reflect the robustness of the model in relation to changes in these parameters.

We set the cancellation cost, $c_{\text {canc }}$, equal to 0.1 in ticks; nevertheless, in analyses presented in Section 9, we modify the cancellation cost to observe the impact of such a measure on the market and the behaviour of the different agents. ${ }^{22}$ In addition, similar

\footnotetext{
22 The value assumed for the cancellation cost is consistent with the value imposed in NYSE Euronext, in which above an order-trade ratio of 100:1 a charge of $€ 0.10$ fee is applied to cancellations. Suppose that a trader in NYSE Euronext submits and cancels 100 consecutive orders and, immediately after that, she submits and cancels another one. The cancellation fee for the 101st cancellation can be shared with the previous uncharged cancellations to 'distribute' the cost, which makes a cancellation cost per order of $€ 0.001$ (i.e. $€ 0.10 / 101=€ 0.001$ ). The cancellation cost per order of $€ 0.001$ represents an under bound since after the 101st cancellation, additional cancellation costs (from unexecuted limit orders) will be divided by the first 100 uncharged cancellations plus the new charged cancellations. Therefore, if we assume that the tick size is $€ 0.01$ our cancellation fee of 0.1 ticks (i.e. $€ 0.001$ ) is similar to what we can observe currently in the market.
} 
to Goettler et al. (2009), we assume that the 'delaying' cost, $\rho_{d}$, is reflected in a continuous discount rate equal to 0.05 for all agents. ${ }^{23}$ In unreported analyses, we experimented with different values for $\rho_{d}$ and obtained results qualitatively equivalent to the ones presented in the following sections.

Even though slow traders may use sophisticated computers to process data and may have a big research team to support decisions, we assume that a trading decision has to pass through a human being who has to read, to understand, to learn, and 'to push the button'. Thus, we assume that the time lag in which slow traders observe the fundamental value of the asset, $\Delta_{t}$, is equal to 1.3 seconds. Similar to the other parameters, we examine the robustness of the model with different values of $\Delta_{t}$; these results are also presented in the following sections.

\section{The effect of HFT on microstructure noise and errors in slow traders' beliefs}

A systematic investigation into the consequences of HFT for market functioning which takes into account the most important features of HFT technology is highly relevant, not only from an academic point of view, but also from a policy perspective. For instance, in 2010, the SEC stated that "By any measure, high frequency trading is a dominant component of the current market structure and is likely to affect nearly all aspects of its performance". In this section, we start the analysis with the effects of HFT on market quality by studying microstructure noise and errors in slow traders' beliefs.

The microstructure features of financial markets may induce some friction that makes the transaction price, $p_{t}$, depart from the fundamental value of the asset, $v_{t}$. Therefore, the transaction price can be decomposed into two components: the fundamental value of the asset plus microstructure noise, $p_{t}=v_{t}+\xi_{t}$, where $\xi_{t}$ is known as

${ }^{23}$ Foucault et al. (2005) also use a similar 'delaying' cost which is called an 'impatience' rate in their study. 
microstructure noise. ${ }^{24}$ In frictionless markets, microstructure noise should be zero; however $\xi_{t}$ can be an important component of prices in real markets.

One important point of friction in financial markets is the limited cognition of market participants. Currently, there is a large amount of information that has to be analysed by agents in order to make optimal trading decisions. However, the analysis of all this information is not perfect due to cognition limits because, for instance, investors can be busy completing other tasks.

HFT may mitigate the cognitive limits of human beings, as computers can rapidly analyse and process a large amount of information. HFT traders have superior speed in processing news and signals, which can be quickly used in trading strategies by the low-latency transmission of orders. Prices should immediately reflect the improvements in the informational analysis. Therefore, HFT may improve informational efficiency by reducing the difference between the fundamental value and the transaction price, and hence decreasing microstructure noise.

Observation 1. Microstructure noise is reduced by the presence of participants with HFT.

In order to reach Observation 1, we present levels of microstructure noise in a scenario with HFT (slow and fast traders in the market) and in a scenario without HFT (only slow traders in the market) in column 2 and column 3 of Table 1. Column 2 and column 3 report the mean of the absolute value of the difference between $v_{t}$ and $p_{t}$ and its standard deviation, respectively. The market is observed every 10 minutes to obtain the values in this table. In Table 1 we can observe reductions in the absolute value and the standard deviation of microstructure noise of $78.23 \%$ and $75.79 \%$, respectively, from the scenario without HFT to the scenario with HFT in the market.

[Insert Table 1 here]

Our results are supported by previous empirical studies, which present evidence that high frequency traders can submit orders in the same direction as price movements

${ }^{24}$ See Hasbrouck (2002) for a discussion of microstructure noise. 
due to the speed advantages that they have in analysing information (e.g. Kirilenko et al., 2011). Hendershott and Riordan (2010) and Brogaard et al. (2012) show that HFT plays a beneficial role in price discovery, as fast investors trade in the direction of permanent price movements, and in the reverse direction of transitory pricing errors. In addition, Brogaard (2010) finds that high frequency traders add more to price discovery than non-HFT agents. ${ }^{25}$

The reduction in microstructure noise due to the trading activity of HFT participants also has an informational impact on the slow traders' beliefs. Slow traders are eager to capture and to learn from any information disclosed in trades, with the objective of improving the accuracy of their estimations about the fundamental value. They do so to make better trading decisions and to decrease the adverse selection that they face when there are fast traders in the market.

Observation 2. The learning process followed by slow traders reduces their belief errors regarding $v_{t}$ in the presence of HFT participants.

Table 1 column 4 and column 5 show the errors in slow traders' beliefs regarding the current fundamental value of the asset. The errors in slow traders' beliefs is defined as $\mathrm{E}\left(v_{t}\right)-v_{t}$, where $\mathrm{E}\left(v_{t}\right)$ reflects their estimations about $v_{t}$. Column 4 and column 5 report the mean of the absolute value and the standard deviation of $\mathrm{E}\left(v_{t}\right)-v_{t}$. Table 1 shows that the errors in beliefs decrease when there are HFT traders in the market. Table 1 reports that there are reductions of $85.47 \%$ and $80.64 \%$ in the absolute value and in the standard deviation of the errors in slow traders' beliefs, respectively, from the case without HFT to the case when HFT participants are also present. Recall that in the scenario with only slow traders there is nothing to learn, since there are no agents with informational advantages. An accurate estimation of the fundamental value is crucial for slow traders to determinate the expected payoff for potential trading strategies in the presence of HFT. For instance, in Section 8 we will show how important this learning process on the realized payoff of slow traders.

\footnotetext{
25 In addition, the reductions in microstructure noise can be related to reductions in transaction price volatility since $p_{t}=v_{t}+\xi_{t}$, as reported in the empirical analysis of Hasbrouck and Saar (2012).
} 
As we mentioned in the introduction, HFT agents have speed advantages in terms of analysing information (informational advantage) and trading submission (the lowlatency transmission of orders and the quick revision of previous trading decisions). Therefore, in Table 2 we examine the effect of these speed advantages on the informational measures reported in Table 1. Table 2 Panel A shows the effects on informational efficiency of different lag periods $\Delta_{t}$ in which slow traders can observe the fundamental value of the asset. Table 2 Panel B reports a similar analysis to Panel A, but it examines diverse levels in the trading speed differences between slow and HFT traders regarding the latency of submissions and modifications regarding unexecuted limit orders.

Observation 3. The informational advantage and trading speed advantage are essential to the improvement in market informational efficiency due to HFT.

As expected, Table 2 Panel A shows that the informational efficiency is reduced as the size of the lag period in which slow traders observe $v_{t}$ increases. In Panel $\mathrm{A}$ microstructure noise, $p_{t}-v_{t}$, increases as the informational lag grows. It is important to notice in Panel A that, since there are no changes in the difference in submission speed between slow and fast traders (which is reported below in Panel B), the informational lag mainly affects the informational efficiency of trades coming from slow traders. This mean that trades coming from fast traders are still informative, which is not the case for slow traders when $\Delta_{t} \neq 0$. Therefore, Panel A shows that an important contribution to microstructure noise comes from the limited cognition of slow traders. The cognition limits of slow traders are reflected in their errors in beliefs about the fundamental value of the asset, $\mathrm{E}\left(v_{t}\right)-v_{t}$, which also increases as the informational lag grows.

\section{[Insert Table 2 here]}

The findings of Table 2 Panel B are consistent with the results of Panel A. Panel B reports that informational efficiency decreases as HFT agents, who are better informed than others, trade faster than slow traders. Microstructure noise is reduced by two effects: first, when the HFT agents trade at higher speed there more 'informed' 
transactions in the market, which diminishes microstructure noise; and second, since there are more informed trades from fast traders, slow traders can use them to improve the accuracy of their estimations about the contemporaneous value of $v_{t}$, thus their trading decisions are based on more precise information, which also reduces microstructure noise. The last effect, improvements in the accuracy of slow traders regarding $\mathrm{E}\left(v_{t}\right)$, is observed in the reduction of the slow trader's errors in beliefs when HFT traders have trading speed advantages regarding slow traders.

\section{The effects of HFT on the submission behaviour of market participants}

In a limit order market, there are liquidity demanders and liquidity suppliers. Liquidity suppliers post limit orders which will be executed later by liquidity demanders through market orders. On the one hand, liquidity demanders have some intrinsic benefits to immediately trade (i.e. alternative benefits to the potential profits from the trading activity per se). On the other hand, liquidity suppliers mainly profit from trading opportunities in the market, and they do not necessarily need to trade right now. In our model, therefore, traders with private values other than zero (i.e. $|\alpha| \neq 0$ ) are likely to be liquidity demanders; while traders with private values equal to zero (i.e. $\alpha=0$ ) are likely to be liquidity suppliers.

Observation 4. HFT induces changes in the submission behaviour of slow traders due to their disadvantages in analysing information and quickly modifying previous trading decisions, which increase the 'picking-off risk' of limit orders that is especially high in the presence HFT traders.

We reach Observation 4 through the results presented in Table 3. Table 3 reports the percentage of order types (market or limit orders) per trader differentiated by private values when there are slow and fast traders in the market. We combine the results for positive and negative private values given that the model is symmetric on both sides of the book. Table 3 shows that traders with a high intrinsic value to trader (i.e. $|\alpha|=8$ ) are willing to submit more market orders than limit orders. Conversely, traders with no additional private benefits are eager to trade more limit orders than 
market orders. However, there is an interesting trading behaviour when the private value is in the middle. Slow traders tend to submit market orders while HFT traders prefer to submit limit orders when $|\alpha|=4$. The intuition behind this result is related to the high 'picking-off risk' that slow traders face when they submit limit orders in an environment with HFT traders. A limit order is a commitment made today to trade the asset at any point in the future at a pre-specified price $p$; thus limit orders can be 'picked-off' by quick investors when market conditions change unfavorably. Slow traders have more difficulty in analysing information and quickly modifying previous trading decisions than fast traders, thus the 'picking-off risk' of limit orders is particularly high for 'traditional' slow agents when there are HFT traders in the market.

\section{[Insert Table 3 here]}

Moreover, Table 3 shows that slow traders have a tendency to submit more market orders than fast traders for each of the private values, which is also explained by the high 'picking-off risk' that slow traders face when they submit limit orders in an environment with HFT. The probability that a trader submits a market order instead of a limit order is higher for slow traders than fast traders at 85.88\%, 153.84\%, and $15.68 \%$ when $\alpha=0,|\alpha|=4$, and $|\alpha|=8$, respectively. The 'picking-off risk' has the largest impact on the behaviour of slow traders when $|\alpha|=4$ because, as explained above, this risk gives additional incentives to slow traders to reduce the submission of limit orders.

Table 4 confirms the evidence provided in Table 3 regarding the change in the trading behaviour of slow traders. Table 4 shows the percentage of order types per trader in the scenarios with and without HFT traders in the market. In the scenario in which slow and fast traders coexist, the probability that a trader submits a market order instead of a limit order is higher, at $177.53 \%$, for slow traders than fast traders. Moreover, Table 4 shows that the presence of HFT traders increases the effect of the 'picking-off risk' on the behaviour of slow traders. In the scenario without HFT in the market, the probability for slow traders of submitting a market order instead of a limit 
order is $33.81 \%$, but this probability increases to $47.58 \%$ (an increment of $40.72 \%$ ) when there are also fast traders competing.

\section{[Insert Table 4 here]}

Table 4 also shows that HFT traders are liquidity suppliers. The probability for a fast trader of submitting a limit order rather than a market order is $82.85 \%$. Therefore, quotes (i.e. bid and ask prices), which are a product of limit orders, should be informative since HFT traders have informational advantages. In fact, in Section 7 we will show that the bid-ask spread decreases when there is HFT in the market.

In relation to the advantages of HFT technology, Table 5 Panel A presents the impact on the type of orders submitted by agents of different lag periods $\Delta_{t}$ in which slow traders can observe the fundamental value of the asset. Panel A shows that the probability that a slow trader submits a market order instead of a limit order increases as the informational lag grows. This probability rises from $39.32 \%$ when slow and fast traders observe at the same time $v_{t}$ to $48.09 \%$ when $\Delta_{t}$ is equal to 2.6 seconds. Table 5 Panel B reports the effects on the type of orders submitted by traders of diverse levels in the trading speed differences between slow and HFT traders concerning the latency of submissions and modifications regarding unexecuted limit orders. Panel B also shows that slow traders submit more market orders when trading speed differences increase. The results of Panel A and Panel B are explained by the 'picking-off risk' increase for slow traders, when both the informational advantage and the trading speed advantage of HFT traders grow.

\section{[Insert Table 5 here]}

Table 5 Panel A and Panel B also report a small increase in the probability that a fast trader submits a market order in place of a limit order, although this increment is not due to their risk of being 'picked-off'; but is rather the opposite. Since HFT traders have an informational advantage they can find trading opportunities in limit orders from slow traders, and can 'pick' these orders through the submission of quick market orders. For instance, in unreported results we find that HFT traders with no private 
values (i.e. $\alpha=0$ ), who are willing to submit limit orders, submit more market orders when there are additional informational and trading speed differences.

Observation 5. The changes in order types submitted by slow traders is caused by differences between slow and HFT traders in terms of information and trading speed, but the latter has a larger impact on the trading behaviour of non-HFT agents.

It is interesting to compare the results from Table 5 Panel $\mathrm{A}$, when $\Delta_{t}$ is equal to zero, with the results of Table 5 Panel $B$, when traders are equally fast in trading speed. Panel B shows that when slow and fast traders have the same trading speed (no trading speed advantage), slow traders have the lowest probability of submitting market orders (22.27\%). This probability for slow traders is $43.35 \%$ lower than the case in which $\Delta_{t}$ is equal to zero (no informational advantage). Indeed, the behaviour of slow traders in order submissions is closer to the behaviour of HFT traders when both have the same speed. Therefore, the changes in the submission behaviour of slow traders, due to the 'picking-off risk' of limit orders, is mainly affected by the advantages of HFT traders in terms of the low-latency transmission of orders and the quick revision of previous trading decisions rather than informational differences.

\section{The impact of HFT on the bid-ask spread and limit order book depth}

What is the effect of HFT on the bid-ask spread and on the depth of the limit order book? In the previous section, we observe in Table 4 that fast traders are likely to submit limit orders since they have informational and trading speed advantages over other agents (i.e. they are mainly liquidity suppliers). Therefore, the bid-ask spread and the depth of the limit order book are directly affected by the trading activity of HFT players.

Observation 6. HFT reduces the bid-ask spread and improves the depth of the limit order book. 
Table 6 examines the effect of HFT on bid-ask spread and the number of limit orders at the ask price. Table 6 column 2 shows that the bid-ask spread is reduced by $67.33 \%$ when non-HFT and HFT traders coexist in comparison to the scenario when there are only slow traders in the market. The reduction in the bid-ask spread is due to three main reasons. First, there is more trading activity in the market when there are HFT traders, which induces a liquidity improvement (i.e. it is easy to find a counterpart for orders). Second, quotes are 'more informative' since HFT traders are willing to submit more limit orders than slow traders, as we explained in the previous section. Third, the quotes that come from the limit order submissions of slow traders are also 'more informative' when there are HFT traders in the market, because slow traders can capture and learn from the information revealed by the fast traders' activity.

\section{[Insert Table 6 here]}

Table 6 column 3 shows that there is an increase in the number of limit orders at the ask price thanks to HFT, since fast traders are willing to exploit their speed advantages through limit orders. The number of limit orders at the ask price increases by $102.92 \%$, when there are slow and fast traders in the market compared to the case when there are only slow traders. The average depth of the book is very small when there are only slow traders, because they prefer to submit market orders rather than limit orders, which is also congruent with the trading behaviour of slow traders described in the previous section.

Our results are consistent with the findings presented in empirical studies, in which HFT is associated with improvements in market liquidity. For instance, Hendershott et al. (2012), Hasbrouck and Saar (2012) and Riordan and Storkenmaier (2012) report that HFT reduces spreads; while Hasbrouck and Saar (2012) also find an increase in the limit order book depth due to the presence of HFT traders.

We additionally analyse in Table 7 the effects of the speed components of HFT on the bid-ask spread and the limit order book depth. Panel A shows that the bid-ask spread has an 'asymmetric information' component. Panel A reports that the bid-ask spread rises as the lag period $\Delta_{t}$, in which slow traders can observe the fundamental value of 
the asset, increases. This is congruent with the microstructure literature in which asymmetric information is positively related to the bid-ask spread (See, e.g., Copeland and Galai, 1983; Glosten and Milgrom, 1985; and Kyle, 1985). In addition, the number of limit orders at the ask price declines as $\Delta_{t}$ grows, which is consistent with Table 5 Panel A, where slow and fast traders decrease the submission of limit orders as the informational lag grows.

Observation 7. The bid-ask spread reductions are due to a drop in the levels of asymmetric information in the market and an increase in market liquidity thanks to HFT technology.

Table 7 Panel B presents evidence that the bid-ask spread also has a liquidity component. Panel B shows that the bid-ask spread decreases and the limit order book depth rises as the trading speed of HFT traders is higher than slow traders. When HFT traders are faster than other market participants, more trading activity comes from the HFT technology, which implies more informative and aggressive quotes combined with more limit orders in general. As a result, there are more chances to find a counterpart for orders, which improves market liquidity.

[Insert Table 7 here]

\section{The payoffs due to trading when there are HFT traders}

What are the economic benefits of HFT? Does HFT generate economic damage for traditional traders? On the one side, a significant amount of money has to be paid to acquire HFT technology since it requires the purchase of computational equipment, subscriptions to real time data providers, highly trained professionals to design HFT algorithms, IT teams to develop connections and platforms, and co-location services to rent a space in the exchange for computers to submit faster orders than competitors. ${ }^{26}$

\footnotetext{
26 The purpose of co-location services provided by exchanges is to decrease the time between order submission by HFT computers and order reception in the exchange servers; and hence to further reduce trading latency.
} 
Therefore, investment in this technology should be justified by an increase in the trading profits of HFT traders. ${ }^{27}$

On the other side, there is some consensus among academics and practitioners regarding the potential economic detriment to trading benefits for slow traders due to the presence of HFT traders. For instance, Biais et al. (2012a) show evidence that when some traders become fast there is an increase of adverse selection costs for the others, thus HFT may generates negative externalities. In addition, in a debate carried out by The Economist on March 7th 2012 Seth Merrin (founder and CEO, Liquidnet) stated that: "High-frequency traders are, by design, trading ahead of market orders, to the detriment of long-term investors. HFT benefits very few, at the expense of very many, which defies the purpose of why a market exists and, as a result, has lessened the overall quality of the markets".

In order to examine the motivation of some agents to invest in HFT technology and the effects of HFT on the profits of slow traders, we calculate the average payoff (i.e. the surplus or utility) for the different agents. Suppose a trader with private value $\alpha$ submits a limit order to buy at price $\tilde{p}_{0}$, which is not modified until the execution at time $t$. The realized payoff for this trader is given by $e^{-\rho_{d} t}\left(\alpha+v_{t}-\tilde{p}_{0}\right)$. Therefore, realized payoffs take into account the private value of the trader. In the case that the same trader submits a market order to buy, the realized payoff is $\left(\alpha+v_{0}-\tilde{p}_{0}\right)$. In the case that the trader submits a limit order and after a while re-enters and cancels the order, then the payoff is given by the same logic as described in equation (1). ${ }^{28}$

Observation 8. It is true that HFT induces some economic damage for slow traders in the case where both fast and slow traders coexist; but it also true that the economic benefits for slow traders are higher after the introduction of HFT technology.

\footnotetext{
27 On July 4, 2007, an article in the International Herald Tribune, entitled "Citigroup to expand electronic trading capabilities by buying Automated Trading Desk" wrote: "Goldman spends tens of millions of dollars on this stuff. They have more people working in their technology area than people on the trading desk...The nature of the markets has changed dramatically".

28 Similar examples can be described for sell orders, but in an opposite direction.
} 
Table 8 presents the average payoff for diverse types of traders differentiated by private values, in the scenarios with HFT and without HFT. In the scenario in which HFT and slow traders are present, Table 8 shows that HFT traders always have higher payoffs than slow traders. In the last column of the table, we can observe that in terms of profits, HFT traders make 3.809 ticks while slow traders make 3.614 ticks. It is important to notice that this difference is on average per order; thus the total trading payoff is much larger since HFT traders submit many more orders than slow traders. Consequently, as in Biais et al. (2012a), we show evidence that HFT traders generate adverse selection costs for other participants. ${ }^{29}$

\section{[Insert Table 8 here]}

Nevertheless, it is also important to answer the following question: Is there any economic gain that HFT may 'indirectly' generate in the profits of slow traders due to improvements in market quality? To answer this question, Table 8 shows the average payoff for slow traders prior to and after HFT. In the last column of Table 8, we can observe that there is an economic improvement in slow traders' trading profits when there are HFT traders, in comparison to the scenario when this technology does not exist. The average payoff for slow traders improves from 2.698 to 3.614 ticks before and after HFT. It interesting to notice that the average payoff increases for the majority of slow traders, with the exception of slow traders with a private value equal to zero (i.e. with $\alpha=0$ ). This is explained by the trading habits of this type of slow trader. Slow traders with no intrinsic value to trade submit more limit orders than market orders (see Table 3); thus they are the ones who have the highest cost due to the 'picking-off risk' that is exacerbated when there are HFT traders in the market. Therefore, when there are no fast traders, the cost associated with the 'picking-off risk' substantially decreases. Thus, the reduction of 'picking-off risk' costs when there are no HFT traders increases the payoffs for slow traders with $\alpha=0$.

\footnotetext{
${ }^{29}$ Despite the profits generated by HFT for some traders who have access to resources to invest in this technology, other agents may not be able to afford such a large initial investment (e.g. small investors or long-term traders who are not trading constantly, and thus they cannot justify such investment). However, agents who decide to be 'slow' can anticipate that HFT traders may be present in the market, and hence they can trade strategically to take this into account. This is consistent with the findings of Biais et al. (2012a), who show that in some scenarios fast and slow traders may co-exist.
} 
The economic improvement in the payoff for slow traders after the introduction of HFT technology is explained by two main factors. First, slow traders have superior information for making better trading decisions due to their cognitive capacity to capture and to learn from the information revealed by HFT traders in the market activity. Second, there is an improvement in market quality reflected in more chances to find a counterpart for an optimal trading strategy given there is a higher limit order book depth together with a lower bid-ask spread. To examine these two factors in the slow traders' payoff, Table 9 shows the same analysis as Table 8, but in Table 9 the learning capacity of slow traders is removed. Table 9 last column shows that the average payoff for slow traders decreases to 3.408 ticks when they do not have the cognitive capacity, compared to the same value in Table 8 (3.614 ticks) where slow traders learn from the information disclosed by HFT traders in the market. Thus, the reduction in asymmetric information due to the learning process followed by slow traders is an important component of their higher profits after HFT. Nevertheless, even without the learning capacity of slow traders, Table 9 shows that their average payoff is still higher than in the scenario when there are no fast traders in the market. Therefore, the improvement in market quality due to HFT is also an important factor that affects the growth of the payoff for slow traders.

\section{[Insert Table 9 here]}

What are the impacts of the HFT speed advantages on the average payoff for the different agents? Table 10 presents the effects of the informational advantage (third column) and the trading speed advantage (fourth column) of HFT on the average payoff for slow and fast traders. Panel A reports the scenarios in which slow traders have cognitive capacities; while in Panel B their learning skills are removed. As expected, Panel A third column shows that when there are no informational advantages the average payoff increases for slow traders and decreases for fast traders. However, the economic benefits for fast traders are still higher (3.798 ticks) than the economic benefits for slow traders (3.636 ticks), which means that trading speed difference among traders should be an important element that affects the agent's payoff. 
Observation 9. The effect of the informational advantage of HFT technology does not have as large an impact on the economic differences between slow and fast traders as the trading speed advantage when slow traders have learning capacity.

In Table 10 Panel A in the fourth column, we observe that in the case that all traders are equally fast in trading speed, the difference in payoff is marginal between slow and fast traders (3.887 ticks and 3.891 ticks, respectively). This result is consistent with the results reported in Section 6 in Table 5 Panel B (column 2 and column 3), where we showed that the submission behaviour of slow traders is similar to the behaviour of fast traders when all traders have the same trading speed. This implies that the effect of the informational advantage of HFT technology does not have as large an impact on the economic differences between slow and fast traders as the trading speed advantage. The reduced difference in payoff between slow and fast traders in Table 10 Panel A (column 4) and the similar submission behaviour of traders in Table 5 (column 2 and column 3) is explained by the 'picking-off risk' costs. The 'picking-off risk' costs are small when traders are equally fast, since slow traders can react as quickly as HFT traders to unfavourable changes in the market conditions.

\section{[Insert Table 10 here]}

The results presented in Table 10 Panel A (column 4) change if we remove the learning capacities of slow traders, as presented in Panel B. Table 10 Panel B (column 4) shows that the learning process followed by slow traders affects their economic benefits; the average payoff for slow traders is reduced (3.752 ticks) in Panel B in comparison to Panel A, while the average payoff for fast traders increases (3.967 ticks).

\section{$9 \quad$ Policy analysis}

An important characteristic of our study is that we can evaluate the impact of possible market regulations and potential changes or shocks on market conditions, as the model allows the simulation of a true order market book in a controlled environment. 
In this section, we consider two policy exercises. We examine the impact of different cancellation fees and different volatility shocks on market quality and stability.

\subsection{The effect of different cancellation fees on HFT}

HFT technology may induce the use of unethical trading practices. For instance, HFT traders can submit a large number of limit orders that are cancelled in a very short period, which is called 'quote stuffing' (see, e.g., Egginton et al., 2012). The main objective of 'quote stuffing' is to flood the book with a high number of fictitious and uninformative orders, and thus to obscure current market conditions for competitors. This affects slow traders in particular, since they are not able to process a large amount of information quickly. In addition, 'quote stuffing' also induces congestion in the IT networks of exchanges, which may generate a collapse of the systems.

Some exchanges have already implemented regulations, such as a cancellation fee, to reduce 'quote stuffing' strategies (e.g. NYSE Euronext); while others argue that additional transaction costs could induce more disadvantages than advantages (e.g. the London Stock Exchange or BATS Chi-X Europe). For instance, in 2011 the London Stock Exchange stated, referring to cancellation fees, that: "Any measure that increases transaction costs will reduce liquidity in markets, increasing the cost of capital for companies, and may also exacerbate volatility".

Although our model cannot characterize 'quote stuffing' strategies since traders can only submit one order, we would like to observe the impact of diverse transaction cancellations on market quality. Table 11 reports the effect of different cancellation fees on the percentage of cancellations per number of traders. Table 11 shows that a cancellation fee effectively reduces the number of cancellations in the market. As expected, the number of cancellations decreases for both slow and fast traders. The probability that an agent makes a cancellation decreases from 35.49\% (73.97\%) when there is no cancellation fee to $19.75 \%(46.13 \%)$ when the cancellation fee is equal to 0.2 ticks for slow traders (fast traders). In addition, we can observe that HFT traders 
cancel more than slow traders, which is consistent with the results of Table 4 (Section 6) since HFT traders submit more limit orders than 'traditional' slow traders.

Observation 10. A cancellation fee reduces the number of cancellations by both HFT and non-HFT traders, but it also negatively affects market quality.

[Insert Table 11 here]

Table 12 shows changes in market quality measures due to different cancellation fees. Table 12 shows that there is a reduction in market informational efficiency when a cancellation fee is applied. A cancellation fee represents additional market friction that affects informational flows. Microstructure noise, $p_{t}-v_{t}$, increases in its absolute value and standard deviation. The slow trader's errors in beliefs, $\mathrm{E}\left(v_{t}\right)-v_{t}$, also increase in both the absolute value and standard deviation, because the cancellation fee generates additional noise in the information that is revealed by the market activity of HFT traders. Thus, Table 12 presents evidence that a cancellation fee can increase the level of asymmetric information between slow and HFT traders. The results of potential damage to the informational efficiency of the market due to a cancellation fee have important policy implications, since the main purpose of a cancellation fee is to generate exactly the opposite result. A cancellation fee is designed to reduce the flood of uninformative limit orders that are cancelled in a short period (i.e. 'quote stuffing'), which obscures market conditions for the rest of the market participants. Instead, Table 12 shows that a cancellation fee can also induce informational damage for traditional slow traders which should be taken into account for the design of regulations.

\section{[Insert Table 12 here]}

Table 12 also shows that the number of limit orders also declines as the cancellation fee grows since some limit orders have a high chance of being cancelled. Moreover, the bid-ask spread increases as the cancellation fee rises, which is due to three main reasons. First, there is less market liquidity, which is reflected in a reduction in the number of limit orders at the ask price when the cancellation fee grows (i.e. it is more difficult to find a counterpart). Second, in unreported results we found that the 
number of limit orders coming from HFT traders decreases, therefore quotes are less informative. Third, we observe that slow trader's errors in beliefs, $\mathrm{E}\left(v_{t}\right)-v_{t}$, also rise, as there are higher cancellation fees (i.e. there is more asymmetric information between slow and fast traders).

Furthermore, Table 12 presents evidence that the global economic benefits of trading are reduced as the cancellation fee grows (see last row). The average payoff for fast traders decreases; however the average payoff for slow traders increases, although marginally. The reason for the improvement in the economic benefits for slow traders when there is a cancellation fee is also related to a reduction in 'picking-off risk' costs of limit orders for slow traders. If a slow trader submits a limit order and the market condition changes unfavourably when there is a cancellation fee, a fast trader with other previous limit orders in the book has to decide between: cancelling her previous order to 'pick' the limit order of the slow trader through a new market order; or keeping her original limit order to avoid the cancellation fee. This decision process by HFT traders induces a reduction in the 'picking-off risk' for slow traders, and hence improves their economic benefits.

\subsection{The effect of diverse volatility shocks on market quality and stability}

There has been intense debate as to whether HFT can affect market stability given the large trading volume generated by high frequency traders. For instance, On September 22, 2010, the SEC Chairman, Mary Schapiro, said that "...high frequency trading firms have a tremendous capacity to affect the stability and integrity of the equity markets. Currently, however, high frequency trading firms are subject to very little in the way of obligations, either to protect that stability by promoting reasonable price continuity in tough times, or to refrain from exacerbating price volatility". This debate was particularly fervent after a brief period of extreme intraday volatility in the U.S. financial market, on May 6, 2010, which is commonly called the 'flash crash'. ${ }^{30}$ Initially, this phenomenon was attributed to a downward spiral of orders from high

30 This volatility was reflected in U.S. stock market indices, stock index futures, options and exchangetraded funds. 
frequency traders. ${ }^{31}$ However, Kirilenko et al. (2011) provide evidence that a wrongly executed selling plan from a large fundamental trader triggered this event. Nevertheless, Kirilenko et al. (2011) state that "During the Flash Crash, the trading behaviour of HFTs appears to have exacerbated the downward move in prices".

Consequently, in this subsection we analyse the effects of unexpected shocks on the volatility of the fundamental value of the asset. These unexpected shocks are performed as follows. In all our analyses and scenarios in this paper, we firstly solve the Markov-perfect equilibrium of the model using the Pakes and McGuire's (2001) algorithm. Let's call this first step of obtaining the model equilibrium: Stage I. This algorithm, as explained in Section 4, can be viewed as a learning process followed by different traders in relation to the trading game described in the model. Therefore, when we obtain the model equilibrium, we can say that agents have 'learned enough' to make optimal decisions in each state given the different characteristics and advantages of traders. Afterwards, we fix agents' beliefs and simulate time series of orders and trades through the model to generate a historical limit order book. Let's call this second step of simulations: Stage II.

All the results presented in this study up to this point were obtained following Stage I and Stage II sequentially with exactly the same parameters. Instead, suppose that in Stage I we obtain the model equilibrium with a volatility of the fundamental value of the asset $\sigma$; we then fix agents' beliefs but in Stage II we simulate orders and trades with a higher volatility $\sigma+\Delta \sigma$. The modification of the model's volatility from Stage I to Stage II implies an unexpected change in market volatility for traders. Traders learned to behave in relation to a volatility $\sigma$ in Stage I, but in the simulations in Stage II they face a volatility $\sigma+\Delta \sigma$ that is unexpected. The increase in the level of volatility from Stage I to Stage II represents an unexpected shock in volatility for the market participants.

\footnotetext{
31 For instance, a perception survey conducted by Market Strategies International, between June 23, 2010 and June 29, 2010, shows that $46 \%$ of 380 retail advisors believed that HFT was the biggest contributor to the 'flash crash', while $83 \%$ of the sample believed HFT was an important contributor.
} 
Observation 11. HFT traders may have incentives to manipulate market volatility since they can make larger profits through limit orders when a volatility shock is applied, which may affect market stability because a limit order market is a 'volatility multiplier'.

Table 13 shows the effect of different unexpected volatility shocks on market quality and stability. The second column is our base case when there is no volatility shock since in our model $\sigma=0.50$ (i.e. $\Delta \sigma=0$ ). The third and fourth columns report volatility shocks of 0.10 and 0.20 , respectively (i.e. $\sigma+\Delta \sigma=0.60$ and $\sigma+\Delta \sigma=0.70$ ). Table 13 shows that microstructure noise, $p_{t}-v_{t}$, increases as the volatility shock increases, which means that the volatility of the transaction price grows more than the volatility of $v_{t}$ since $p_{t}=v_{t}+$ (microstructure noise). This is consistent with the results of Goettler et al. (2009), who show that a limit order market is a 'volatility multiplier'.

[Insert Table 13 here]

In Table 13, the slow trader's errors in beliefs, $\mathrm{E}\left(v_{t}\right)-v_{t}$, also increase as the volatility shock increases. This is explained by the additional noise generated by the volatility shock in the information disclosed by HFT traders in their trading behaviour. This noise affects negatively the learning process followed by slow traders. In addition, a volatility shock induces traders to prefer market orders over limit orders, especially for slow traders, since the 'picking-off risk' is higher given that the market conditions can change more drastically. The increase in the 'picking-off risk' also causes a decrease in the number of limit orders at the ask price since traders choose to reduce the number of limit orders. The bid-ask spread is amplified when there is a volatility shock given that there is lower market liquidity and the levels of asymmetric information between slow and fast traders rise.

Table 13 also shows an interesting phenomenon related to the economic benefits of trading. The average payoff for slow traders decreases as the volatility shock grows since they have more chances of being 'picked-off' given that there are larger market changes. However, the average payoff for fast traders increases as the volatility shock 
grows. This is due to the non-linear features of limit orders. Limit orders have a similar structure to option contracts since both are ex-ante commitments to trade in the future at a specified price (see Copeland and Galai, 1983). As with option contracts, the average payoff of a limit order increases as the volatility grows if you can react quickly to market changes, which is the case for HFT traders. Therefore, the average payoff for HFT traders is positively related to the size of the volatility shock. This phenomenon has an important implication, since HFT traders may have incentives to manipulate prices to increase market volatility. Therefore, potential regulations to prevent unfair practices regarding market volatility have to be implemented. This finding is also consistent with Kirilenko et al.'s (2011) study in relation to the 'flash crash', in which they report that the abnormal trading behaviour of HFT traders intensified the market volatility.

In relation to the number of cancellations, as expected, slow traders cancel more limit orders when the volatility shock is higher. In the case of HFT traders, their tendency to cancel limit orders decreases as the volatility shock grows, which can be understood using the same arguments as in the previous paragraph, where we explained that limit orders are more profitable when there is a high level of market volatility. Thus, HFT traders have fewer incentives to cancel their limit orders when the size of the shock increases.

Finally, in unreported results we also examine the impact of different unexpected negative liquidity shocks on market quality and stability. We perform the negative liquidity shocks in a similar way to the volatility shocks. In the case of a liquidity shock, in Stage I we obtain the model equilibrium with a model setup in which trader arrivals follow two Poisson processes with parameters $\lambda^{H F T}$ for fast traders and $\lambda^{S T}$ for slow traders. Therefore, the expected arrival times for HFT traders and slow traders are $1 / \lambda^{H F T}$ and $1 / \lambda^{H F T}$, respectively. However, we simulate orders and trades in Stage II with larger expected arrival times for both slow and fast traders, which is unexpected by market participants. This negative liquidity shock also generates an unfavourable impact on market quality and stability. As expected, when we applied a negative liquidity shock, we found that microstructure noise increases, slow traders' 
errors in beliefs rise, the bid-ask spread grows, the number of cancellations increases, the number of limit orders at the ask price is reduced, and the payoff for all traders decreases. Consequently, regulations to maintain market stability in 'unusual' market conditions are particularly pertinent given the large volume of trading activity generated by HFT participants.

\section{Conclusions}

HFT represents one of the most important market transformations since NASDAQ became the world's first electronic stock market in 1971. Therefore, a systematic study of the effects of HFT on market quality and stability is highly relevant because the potential benefits and dangers of this new technology have not yet been fully understood. In this study, we introduce a dynamic equilibrium model with HFT which describes a stochastic sequential game in a limit order market. HFT traders have superior speed to traditional market participants in terms of analysing information as in Martinez and Roşu (2011), Biais et al. (2012a), and Foucault et al. (2012). However, in our model we also incorporate a trading submission speed advantage for HFT traders that is reflected in the low-latency transmission of orders and quick modifications to previous trading decisions. The model includes the main features of a limit order market, and thus we can generate a historical limit order book which represents an additional contribution of our research.

We find that HFT improves market informational efficiency. HFT reduces microstructure noise and decreases the errors in slow traders' beliefs since they can learn from the information revealed by HFT traders in the market activity. HFT traders are mainly liquidity suppliers since they prefer to submit limit orders; while slow traders prefer to submit market orders to avoid the 'picking-off risk' associated with limit orders. HFT also improves market liquidity given that there is more chance of finding a counterpart for order submissions; this is reflected in an improvement in the depth of the limit order book and a reduction in the bid-ask spread. The reductions in the bid-ask spread are also related to the improvements in market 
informational efficiency, because HFT traders submit more informative quotes and slow traders can learn from them.

We show that HFT traders make larger trading profits than slow traders. However the trading profits for slow traders are higher after the introduction of HFT than before this technology. This is due to the improvement in market quality and reductions in asymmetric information among agents induced by HFT, which indirectly induces an economic gain for slow traders.

We perform two policy exercises using the dynamic features of our model. We find that a cancellation fee reduces the number of cancellations, but also negatively affects market quality. In addition, we show that HFT traders may have incentives to manipulate market volatility since they can make larger profits through limit orders when volatility is high, which may affect market stability because a limit order market is a 'volatility multiplier'.

Finally, the model presented in our paper is intuitive because we include the main features of HFT traders in a dynamic limit order market; however other interesting issues remain to be addressed. For instance, the study of the effect of HFT on the correlations of different assets, the study of the effect on multiple markets, and the analysis when dark pools are present has been left for future research.

\section{Appendix}

The updating process to reach the equilibrium: At any state $s$ of the economy there is a set of possible actions, $\Gamma(s)$, that a trader can follow. Suppose that a given trader arrives for the first time or re-enters at the market at time $t$ and observes the state $s$. In our model setup, the trader has beliefs about the expected payoff of each possible action that could be taken given the observed state $s$. Suppose that $U_{t}(\tilde{a} \mid s)$ is the expected payoff before transaction costs at time $t$ that is associated with the action $\tilde{a} \in \Gamma(s)$. The trader decides to take the optimal action $\tilde{a}^{*}$ that provides the maximum expected payoff after transaction costs out of all possible actions. As a first case, 
suppose that the optimal action $\tilde{a}^{*}$ is not a market order (e.g., a limit order, or a cancellation and resubmission). Later on at time $t_{r}$ the same trader re-enters at the market, but the market conditions have changed. The trader observes a new state $s_{t_{r}}$ in which she follows the optimal strategy $\tilde{a}^{* *}$ that also gives a maximum payoff after transaction costs given the new market conditions. Consequently, the original decision $\tilde{a}^{*}$ induces a realized continuation of optimal actions and expected payoffs; and thus the updating process of beliefs can be written as:

$$
U_{t_{r}}\left(\tilde{a}^{*} \mid s\right)=\frac{n_{\tilde{a}^{*}, s}}{n_{\tilde{a}^{*}, s}+1} U_{t}\left(\tilde{a}^{*} \mid s\right)+\frac{1}{n_{\tilde{a}^{*}, s}+1} e^{-\rho_{d}\left(t_{r}-t\right)}\left(U_{t_{r}}\left(\tilde{a}^{* *} \mid s_{t_{r}}\right)-\tilde{z}_{\tilde{a}^{* *}} C_{c a n c}\right),
$$

where $n_{\tilde{a}^{*}, S}$ is a counter that increases by one when the action $\tilde{a}^{*}$ is taken in the state 5.32

Instead, as a second case, suppose that the optimal decision $\tilde{a}^{*}$ is a market order (i.e. there is no future time $t_{r}$ as in the previous case). Then, the updating expected payoff of this optimal action $\tilde{a}^{*}$ in this scenario can be expressed as:

$$
U_{t}\left(\tilde{a}^{*} \mid s\right)=\frac{n_{\tilde{a}^{*}, s}}{n_{\tilde{a}^{*}, s}+1} U_{t}\left(\tilde{a}^{*} \mid s\right)+\frac{1}{n_{\tilde{a}^{*}, s}+1}\left(\alpha+v_{t}-\tilde{p}\right) \tilde{x}
$$

Here $\tilde{p}$ is the submission price, $\alpha$ is the private value of the trader, $v_{t}$ is the fundamental value of the asset, and $\tilde{x}$ is equal to one (minus one) when the trader submits a buy (sell) order. ${ }^{33}$

As a third case, suppose that the optimal decision $\tilde{a}^{*}$ is a limit order; however later on at time $t_{r}$ this limit order is executed due to the fact that another trader submits a market order. The updating process for the first trader with the optimal action $\tilde{a}^{*}$ can be reflected in the following equation:

\footnotetext{
32 The value of $n_{\tilde{a}^{*}, s}$ affects how quickly we reach the model equilibrium (a large value in $n_{\tilde{a}^{*}, s}$ is associated with a slow convergence). Therefore, we reset $n_{\tilde{a}^{*}, s}$ after a while to improve the convergence speed.

${ }^{33}$ Equation (A2) is also valid for slow traders who do not know the contemporaneous fundamental value of the asset, since they will eventually know it after a lag $\Delta_{t}$ and thus they can also update their beliefs regarding the expected payoffs of their actions.
} 


$$
U_{t_{r}}\left(\tilde{a}^{*} \mid s\right)=\frac{n_{\tilde{a}^{*}, s}}{n_{\tilde{a}^{*}, s}+1} U_{t}\left(\tilde{a}^{*} \mid s\right)+\frac{1}{n_{\tilde{a}^{*}, s}+1} e^{-\rho_{d}\left(t_{r}-t\right)}\left(\alpha+v_{t_{r}}-\tilde{p}\right) \tilde{x},
$$

where $\alpha$ is the private value for the first trader. Similarly, for the second trader who submits the market order that executes the limit order of the first trader, the updating process can be expressed as:

$$
U_{t_{r}}\left(\tilde{a} \mid s_{t_{r}}\right)=\frac{n_{\tilde{a}, s_{t_{r}}}}{n_{\tilde{a}, s_{t_{r}}}+1} U_{t_{r}}\left(\tilde{a} \mid s_{t_{r}}\right)+\frac{1}{n_{\tilde{a}, s_{t_{r}}}+1}\left(\alpha+v_{t}-\tilde{p}\right) \tilde{x}
$$

in which $\alpha$ and $\tilde{a}$ are the private value and the optimal decision of the second trader, respectively. In this case, $\tilde{a}$ is a market order which is chosen at time $t_{r}$ by the second trader when the state $s_{t_{r}}$ is found. It is important to observe in this last case that any market order implies the execution of a previously submitted limit order. Thus, in the presence of market orders the updating process in beliefs always involves two traders: the trader who submits the market order, and the trader who submitted a limit order which is executed by the market order (see equation (A3) and equation (A4)).34

The learning capacity of slow traders: As mentioned previously, even though slow traders can observe the fundamental value of the asset with a time lag $\Delta_{t}$, the model takes into account that slow traders can improve the accuracy of their beliefs regarding $v_{t}$ by observing the market trading activity. Suppose that a slow trader arrives in the market at time $t$. The slow trader observes the fundamental value $v_{t-\Delta t}$, the current limit order book $L_{t}$ that is result of the previous trading activity, the price of the most recent transaction $\hat{p}_{t}$, and the direction of that transaction $\hat{b}_{t}$ which is one

\footnotetext{
34 The initial beliefs about the expected payoffs $U_{0}(\tilde{a} \mid s)$ of the possible actions $\tilde{a} \in \Gamma(s)$ that a trader can take given that she faces state $s$ are set as follows. Suppose one of the possible actions for a trader with private value $\alpha$ in the state $s$ is to submit a limit sell order at price $p$ when the fundamental value is $v$. We set the initial expected payoff of this action as $p-v-\alpha$ discounted by $\rho_{d}$ until the expected time that a new fast trader arrives at the market. This value is only a first approximation since we assume that $v$ is constant, which is not true in the model, and there is a chance that the next trader may submit another limit order instead of a market order that executes the limit order of the previous trader. In the case of a market sell order the expected payoff is simply $p-v-\alpha$ without any discount. Similar values are obtained for buy orders.
} 
(minus one) if the last transaction was the product of a buy (sell) order. ${ }^{35}$ Let $\mathrm{E}\left[v_{t} \mid v_{t-\Delta t}, L_{t}, \hat{p}_{t}, \hat{b}_{t}\right]=v_{t-\Delta t}+\phi\left(v_{t-\Delta t}, L_{t}, \hat{p}_{t}, \hat{b}_{t}\right)$ be the expected value of the fundamental value of the asset given $v_{t-\Delta t}, L_{t}, \hat{p}_{t}$, and $\hat{b}_{t}$, in which $\phi(\cdot)$ is the adjustments that a slow trader has to apply to $v_{t-\Delta t}$ in order to improve the accuracy of her beliefs about $v_{t}$. Let $j\left(v_{t-\Delta t}, L_{t}, \hat{p}_{t}, \hat{b}_{t}\right)$ be the number of times in which $v_{t-\Delta t}, L_{t}$, $\hat{p}_{t}$, and $\hat{b}_{t}$ are observed in the trading game. We suppress the dependent variables in $j$ for notational convenience. Therefore, we can update $\phi(\cdot)$ as follows:

$$
\phi_{j+1}\left(v_{t-\Delta t}, L_{t}, \hat{p}_{t}, \hat{b}_{t}\right)=\frac{j}{j+1} \phi_{j}\left(v_{t-\Delta t}, L_{t}, \hat{p}_{t}, \hat{b}_{t}\right)+\frac{1}{j+1}\left(v_{t}-v_{t-\Delta t}\right)
$$

where $\phi_{0}(\cdot)=0$. Thus, for a slow trader the expected fundamental value is $\mathrm{E}\left[v_{t} \mid \cdot\right]=v_{t-\Delta t}+\phi(\cdot)$, while for a fast trader $\mathrm{E}\left[v_{t} \mid \cdot\right]=v_{t}$.

Convergence criteria: We check for convergence after running the trading game for some billion trading events. Afterwards, we check the evolution of agents' beliefs every 300 million simulations. Suppose that the first group of 300 million simulations after we start checking for convergence finishes at time $t_{1}$. Suppose that the subsequent second group of 300 million simulations finishes at time $t_{2}$. Let $U_{t_{1}}(\tilde{a} \mid s)$ and $U_{t_{2}}(\tilde{a} \mid s)$ be the expected payoffs that are associated with the action $\tilde{a}$ when the state $s$ is present at time $t_{1}$ and $t_{2}$, respectively. In addition, suppose that $k_{\tilde{a}, s}^{t_{1}, t_{1}}$ is the number of times that the action $\tilde{a}$ was taken between $t_{1}$ and $t_{2}$ when traders face $s$. Similar to Goettler et al. (2009), we evaluate the change in the expected value $\left|U_{t_{2}}(\tilde{a} \mid s)-U_{t_{1}}(\tilde{a} \mid s)\right|$ of all pairs $(\tilde{a}, s)$ weighted by $k_{\tilde{a}, s}^{t_{1}, t_{1}}$ every 300 million simulations. Once this weighted absolute difference is smaller than 0.01 (which suggests that the model has converged), we apply other two convergence criteria as Pakes and McGuire (2001) explain in their algorithm. ${ }^{36}$

\footnotetext{
35 Previous transaction prices are implicit in the information of the current limit order book $L_{t}$ which is the outcome of previous trades.

${ }^{36}$ We also induce trembles in the traders' decisions to ensure that the updating process considers all possible actions in each state when we run the trading game to solve the equilibrium. Specifically, we disturb the traders' decisions with a small probability $\xi$ to select actions that are suboptimal while the algorithm converges. We set $\xi$ equal to $0.50 \%$. In the case of a tremble, the trader selects among all suboptimal actions with equal probability. Once we reach the equilibrium of the model, we make $\xi$ equal
} 
After reaching a small weighted absolute difference in the change in the expected values as described in the previous paragraph, we fix the agents' beliefs concerning the expected payoffs, $U^{*}(\cdot)$, and simulate the trading game for other 300 million events. Then, we calculate the realized payoffs of all order submissions after they have been executed. Let $\tilde{J}(\cdot)$ be the realized payoffs. It is important to observe that $\tilde{J}(\cdot)$ is a direct measure of the benefits to trade which is not "averaged" as in equation (A1) to equation (A4). First, we require that the correlation between $U^{*}(\cdot)$ and $\tilde{J}(\cdot)$ is higher than 0.99 . Second, we calculate the mean absolute difference between $U^{*}(\cdot)$ and $\tilde{J}(\cdot)$ weighted by the number of times that a specific action is selected in a given state in the last 300 million simulated events, which also has to be below 0.01 (i.e. in a similar way to the previous paragraph when we evaluate the change in the expected value between $U_{t_{2}}(\tilde{a} \mid s)$ and $U_{t_{2}}(\tilde{a} \mid s)$ weighted by $\left.k_{\tilde{a}, s}^{t_{1}, t_{1}}\right)$. If any convergence criterion is not reached we continue simulating the trading game and updating the beliefs through equation (A1) to equation (A4) until all convergence criteria are satisfied.

Simplifying the state space: In principle, the dimensionality of the state space is unbounded given that the fundamental value of the asset: i) is a continuous variable, and ii) may evolve to diverse levels. The unbounded limits of the fundamental value of the asset also affect the structure of the limit order book (i.e. we could have an infinite grid of prices). Nevertheless, following Goettler et al. (2009), we use some model properties to make the problem computationally tractable by simplifying the state space in the trading game. Firstly, although the fundamental value of the asset follows a random walk, agents do not take into account all the numbers to the right of the decimal separator of $v$ (i.e. agents observe 95.01 instead of 95.0100236). This discretization is in fact what limit order markets do in reality, since the limit order book is a discrete grid of prices with increments of the size of one tick. For instance, the tick size of a stock in NYSE Euronext is equal to $€ 0.01$ if its price is between $€ 50$ and $€$ 99.99. Thus, we create a discrete vector of prices $\left\{\hat{v}^{j}\right\}_{j=0}^{\infty}$ where $\hat{v}^{0}=0$ and $\hat{v}^{j+1}-\hat{v}^{j}=d$, which reflects the levels of the fundamental value that a trader observes, and $d$ is equal to the tick size of the limit order book. Therefore, if the

to zero to simulate a further 300 million events, which are the events that are reflected in the results of the different analyses in our study. 
fundamental value of the asset at a given time is $v$, then the level that a trader views is $\hat{v}^{*} \in\left\{\hat{v}^{j}\right\}_{j=0}^{\infty}$ given by $\hat{v}^{*}-d / 2 \leq v<\hat{v}^{*}+d / 2$.

Nevertheless, the vector $\left\{\hat{v}^{j}\right\}_{j=0}^{\infty}$ still has an infinite set of prices, which means that the limit order book's grid of prices has to be unbounded. For that reason, and secondly, we always put the centre of the book at the discrete level of the fundamental value that a trader observes. Consequently, given that the limit order book is also a discrete set of prices $\left\{p^{i}\right\}_{i=-\infty}^{\infty}$ with a tick size $d$, we put the centre of the book $p^{0}$ equal to $\hat{v}^{*}$ which is defined previously. ${ }^{37}$ For instance, imagine the scenario in which the discrete level of the fundamental value today is $\hat{v}^{*}$ but after a while $v$ changes enough to be closer to $\hat{v}^{* *}$ (i.e. $\hat{v}^{* *}-d / 2 \leq v<\hat{v}^{* *}+d / 2$ ). Suppose that $\hat{v}^{* *}-\hat{v}^{*}=m d$ where $m$ is an integer that can be positive or negative. In this scenario, we move the book in $m$ ticks to centre the book again at the discrete level of the current fundamental value $\hat{v}^{* *}$. In addition, since we move the centre of book, we also modify the existing limit orders in the book to take into account the new relative difference with respect to $\hat{v}^{* *}$. Thus, prices of all orders are always relative to the current discrete level of the fundamental value, which makes traders think about relative prices in relation to $\hat{v}^{* *}$. Moreover, previous transaction prices can be also expressed relative to the current value of $\hat{v}^{* *}$. This allows us to greatly reduce the dimensionality of the state space. ${ }^{38}$

In spite of the reductions in the dimensionality of the state space of the model achieved by the preceding simplifications, the state space is still very large and computationally intractable. This is due to the many possible shapes and features that the order book can present (which, combined with the different types of traders, private values, and beliefs about the fundamental value of the asset, makes the dimensionality of the state space enormous). Ideally, we would like to include all the futures of the market book but this would make the problem unmanageable

\footnotetext{
37 The number of prices around $p^{0}$, that constitute the grid of the book, is selected high enough that even very 'unaggressive' strategies (i.e. with limit orders far away from the fundamental value) can never go outside the grid of prices.

${ }^{38}$ It is important to notice that slow traders and fast traders observe the book differently given their informational differences. Fast traders observe the book centred on the discrete value of $v_{t}$. However, slow traders know $v_{t-\Delta t}$ but they can also observe the trading activity until $t$ to improve the accuracy of their expectations regarding $v_{t}$. Thefreore, slow traders observe the book centred on discrete level of their expected value of $v_{t}$ which also corresponds to one of the prices of $\left\{\hat{v}^{j}\right\}_{j=0}^{\infty}$.
} 
computationally. Consequently, similar to Goettler et al. (2009), we characterize the book with the following combination of variables: i) the current bid and ask prices; ii) the depth at the bid price and the depth at the ask price; and iii) the total depth (including all the orders) on the buy side and the total depth on the sell side.

\section{References}

Alvarez,F, L. Guiso, and F. Lippi, 2012, Durable consumption and asset management with transaction and observation costs, American Economic Review 102, 2272-2300.

Alvarez, F., F Lippi, and L. Paciello,2011, Optimal price setting with observation and menu costs, Quarterly Journal of Economics 126, 1909-1960.

Biais, B., T. Foucault, and S. Moinas, 2012a, Equilibrium high frequency trading, working paper Toulouse School of Economics.

Bruno Biais, Johan Hombert, and Pierre-Olivier Weill. Pricing and liquidity with sticky trading plans. Working paper, TSE, HEC, UCLA, 2012b.

Brogaard, J.A., 2010, High frequency trading and its impact on market quality, working paper Northwestern University.

Brogaard, J.A., Hendershott, T., and R. Riordan, 2012, High Frequency Trading and Price Discovery, working paper UC Berkeley.

Chaboud, A., B. Chiquoine, E. Hjalmarsson, and C. Vega, 2011, Rise of the Machines: Algorithmic Trading in the Foreign Exchange Market, working paper Board of Governors of the Federal Reserve System.

Copeland, Thomas E., and Dan Galai, 1983, Information effects on the bid-ask spread, Journal of Finance 38, 1457-1469.

Cont, R., 2011. Statistical modeling of high frequency financial data: facts, models and challenges. IEEE Signal Processing 28 (5), 16-25.

Egginton, J., B. F. VanNess, and R. A. VanNess, 2012, Quote stuffing, Working paper, University of Mississippi.

Foucault, T., 1999, Order Flow Composition and Trading Costs in a Dynamic Limit Order Market, Journal of Financial Markets 2, 99-134.

Foucault, T., J. Hombert, and I. Roşu, 2012, News Trading and Speed, working paper HEC Paris.

Foucault, T., O. Kadan and E. Kandel, 2005, The Limit Order Book as a Market for Liquidity, Review of Financial Studies 18, 1171-1217.

Glosten, L. R., Milgrom, P. R., 1985. Bid, ask and transaction prices in a specialist market with heterogeneously informed traders. Journal of Financial Economics 14, 71-100.

Goettler, R., C. Parlour, and U. Rajan, 2005, Equilibrium in a Dynamic Limit Order Market, Journal of Finance 60, 2149-2192.

Goettler, R., C. Parlour, and U. Rajan, 2009, Informed traders and limit order markets, Journal of Financial Economics 93, 67-87.

Government Office for Science, 2012, Foresight: The Future of Computer Trading in Financial Markets (2012) Final Project Report, London.

Hasbrouck, J. ,2002. Stalking the "efficient price" in market microstructure specifications: an overview. Journal of Financial Markets 5, 329-339.

Hasbrouck, J., and G. Saar, 2009, Technology and liquidity provision: The blurring of traditional definitions, Journal of Financial Markets 12, 143-172.

Hasbrouck, J. and G. Saar, 2012, Low-latency trading, working paper, AFA 2012 Chicago Meetings Paper. 
Hendershott, T., and R. Riordan, 2010, Algorithmic trading and information, working paper UC Berkeley.

Hendershott, T., C. Jones and A. Menkveld, 2012, Does Algorithmic trading improve liquidity,"Journal of Finance 66 1-33.

Hollifield, B., Miller, R., Sandås, P., Slive, J., 2006. Estimating the gains from trade in limit order markets. Journal of Finance 61, 2753-2804.

Jain, P. K., 2005, Financial Market Design and the Equity Premium: Electronic versus Floor Trading, Journal of Finance 60, 2955-2985.

Jovanovic, Boyan, and Albert J. Menkveld. (2011). "Middlemen in Limit-Order Markets." Working Paper, New York University and VU University of Amsterdam.

Kirilenko, A., A.S. Kyle, M. Samadi, and T. Tuzun, 2011, The Flash Crash: The Impact of High Frequency Trading on an Electronic Market, working paper University of Maryland.

Kleidon, A. W., 1986, Variance bounds tests and stock price valuation models, Journal of Political Economy 94, 953-1001.

Kosinski, R. J., 2012, A literature review on reaction time, Working paper, Clemson University.

Kyle, A.P., 1985. Continuous auctions and insider trading. Econometrica 53, 1315-1336.

London Stock Exchange 2011, LSEG Public Comment to IOSCO's Consultation Report: Regulatory Issues Raised by the Impact of Technological Changes on Market Integrity and Efficiency (CR02/11, July 2011).

Lynch, A. W., 1996, Decision frequency and synchronization across agents: Implications for aggregate consumption and equity return, Journal of Finance 51, 1479-1497.

Mankiw G., and R. Reis, 2002, Sticky information versus sticky prices: a proposal to replace the new keynesian phillips curve, Quarterly Journal of Economics 4, 1295-1328.

Martinez, V., Roşu, I., 2011. High-frequency traders, news and volatility. working paper HEC Paris.

NYSE Euronext, 2012, Trading Fee Guide for NYSE Euronext European Cash Market Members. Trading fee guide for European equities, NYSE Euronext.

Pagnotta, Emiliano, and Thomas Philippon, 2012, Competing on Speed, Working Paper New York University.

Parlour, C., 1998, Price Dynamics in Limit Order Markets, Review of Financial Studies 11, 789816.

Ricardo, R., 2006a, Inattentive producers, Review of Economic Studies 3, 793-821.

Ricardo, R., 2006b, Inattentive consumers. Journal of Monetary Economics 8, 1761-1800.

Riordan, Ryan, and Andreas Storkenmaier, 2012, Latency, liquidity, and price discovery, Journal of Financial Markets 15, 416-437.

Pakes, A., and P. McGuire, 2001, Stochastic algorithms, symmetric Markov perfect equilibrium, and the 'curse' of dimensionality, Econometrica 69, 1261-1281.

Rosu, I. , 2009, A Dynamic Model of the Limit Order Book, Review of Financial Studies 22, 4601-4641.

SEC, 2010, Concept release on equity market structure, Release No. 34-61358, File No. S7-0210.

Trimmel, M., and G. Poelzl. 2006. Impact of background noise on reaction time and brain DC potential changes of VDT-based spatial attention. Ergonomics 49(2): 202-209.

Verrecchia, R., 1982. Information acquisition in a noisy rational expectations economy. Econometrica 50, 1415-1430.

Zhang, Frank, 2010, The effect of high-frequency trading on stock volatility and price discovery,working paper Yale University. 


\section{$\underline{\text { Tables }}$}

\section{Table 1}

Changes in microstructure noise and the slow trader's errors in beliefs due to HFT technology

The table reports microstructure noise and the slow trader's errors in beliefs in the scenarios with HFT (slow and fast traders in the market) and without HFT (only slow traders in the market). Microstructure noise is $p_{t}-v_{t}$, where $p_{t}$ and $v_{t}$ are the transaction price and the fundamental value of the asset, respectively. The errors in beliefs of slow traders, $\mathrm{E}\left(v_{t}\right)-v_{t}$, are regarding the fundamental value of the asset which is observed by slow traders with a lag $\Delta_{t}$. Nevertheless, slow traders can learn from the trading activity of fast traders (who know the contemporaneous value of $v_{t}$ ) to improve the accuracy of their beliefs $\mathrm{E}\left(v_{t}\right)$. The market is observed every 10 minutes. All measures are expressed in ticks. The Markov equilibrium and the posterior market simulation are obtained independently for each case.

\begin{tabular}{|c|c|c|c|c|}
\hline & \multicolumn{2}{|c|}{ Microstructure noise } & \multicolumn{2}{|c|}{$\begin{array}{l}\text { Belief errors of slow traders } \\
\text { regarding the fundamental value }\end{array}$} \\
\hline & Mean $\left|v_{t}-p_{t}\right|$ & Std. Dev. $\left(v_{t}-p_{t}\right)$ & Mean $\left|\mathrm{E}\left(v_{t}\right)-v_{t}\right|$ & Std. Dev $\left(\mathrm{E}\left(v_{t}\right)-v_{t}\right)$ \\
\hline & \multicolumn{4}{|c|}{ Slow traders and fast traders in the market } \\
\hline \multirow[t]{2}{*}{ Slow and Fast traders } & 0.533 & 0.747 & 0.224 & 0.387 \\
\hline & \multicolumn{4}{|c|}{ Only slow traders in the market } \\
\hline Slow traders & 2.449 & 3.085 & 1.542 & 1.999 \\
\hline
\end{tabular}

\section{Table 2}

The effects of speed components of HFT on microstructure noise and slow traders' errors in beliefs

The table presents the effects of the informational advantages and trading speed advantages of HFT traders on microstructure noise $\left(p_{t}-v_{t}\right)$ and the slow trader's errors in beliefs $\left(\mathrm{E}\left(v_{t}\right)-v_{t}\right)$. The variables $p_{t}, v_{t}$, and $\mathrm{E}\left(v_{t}\right)$ are defined in Table 1 and expressed in ticks. The market is observed every 10 minutes. The informational advantages of HFT traders are reflected in different lag periods $\Delta_{t}$ in which slow traders can observe the fundamental value of the asset. The lag periods presented are $\Delta_{t}=0.0 \mathrm{sec}, \Delta_{t}=1.3 \mathrm{sec}$, and $\Delta_{t}=2.6 \mathrm{sec}$. The trading speed advantages of HFT traders are reflected in the different number of times that slow traders are 'slower' than fast traders in latency of submissions and modifications to previous limit orders. The difference in terms of trading speed advantages are performed by modifying the rate of the Poisson processes for the trader's arrivals $\left(\lambda^{H F T}\right.$ and $\lambda^{S T}$ for fast and slow traders, respectively) and the Poisson processes for the traders' re-enters ( $\lambda_{r}^{H F T}$ and $\lambda_{r}^{S T}$ for fast and slow traders, respectively). For instance, when slow and fast traders are equally fast in terms of trading speed $\lambda^{H F T}=\lambda^{S T}$ and $\lambda_{r}^{H F T}=\lambda_{r}^{S T}$, and when slow traders are 40 times 'slower' than traders $\lambda^{H F T}=40 \lambda^{S T}$ and $\lambda_{r}^{H F T}=40 \lambda_{r}^{S T}$. The Markov equilibrium and the posterior market simulation are obtained independently for each combination of parameters.

Slow traders and fast traders in the market

\begin{tabular}{|c|c|c|c|}
\hline & \multicolumn{3}{|c|}{ Panel A:Information lag } \\
\hline & $\Delta_{t}=0.0 \mathrm{sec}$ & $\Delta_{t}=1.3 \mathrm{sec}$ & $\Delta_{t}=2.6 \mathrm{sec}$ \\
\hline Mean $\left|v_{t}-p_{t}\right|$ & 0.499 & 0.533 & 0.547 \\
\hline Std. Dev. $\left(v_{t}-p_{t}\right)$ & 0.718 & 0.747 & 0.757 \\
\hline Mean $\left|\mathrm{E}\left(v_{t}\right)-v_{t}\right|$ & 0.000 & 0.224 & 0.237 \\
\hline \multirow[t]{3}{*}{ Std. Dev $\left(\mathrm{E}\left(v_{t}\right)-v_{t}\right)$} & 0.000 & 0.387 & 0.399 \\
\hline & \multicolumn{3}{|c|}{$\begin{array}{l}\text { Panel B: Number of times that slow traders are slower than HFT traders in trading speed (Low } \\
\text { latency submissions and modifications of previous limit orders) }\end{array}$} \\
\hline & 1 & 20 & 40 \\
\hline Mean $\left|v_{t}-p_{t}\right|$ & 0.696 & 0.533 & 0.530 \\
\hline Std. Dev. $\left(v_{t}-p_{t}\right)$ & 0.973 & 0.747 & 0.743 \\
\hline Mean $\left|\mathrm{E}\left(v_{t}\right)-v_{t}\right|$ & 0.534 & 0.224 & 0.211 \\
\hline Std. Dev $\left(\mathrm{E}\left(v_{t}\right)-v_{t}\right)$ & 0.771 & 0.387 & 0.375 \\
\hline
\end{tabular}


Table 3

Percentage of type of order submission per trader

The table contains the percentage of order types (market and limit orders) per trader differentiated by private values $(\alpha)$. We combine the results for positive and negative private values given that the model is symmetric on both sides of the book. The Markov equilibrium and the posterior market simulation are obtained in the scenario when there are slow and fast traders in the market.

\begin{tabular}{|c|c|c|c|c|c|c|c|c|c|}
\hline & \multicolumn{9}{|c|}{ Slow traders and fast traders in the market } \\
\hline & \multicolumn{9}{|c|}{$\%$ of order types per private values and trader characteristics } \\
\hline & \multicolumn{9}{|c|}{ Private value $|\alpha|$} \\
\hline & \multicolumn{3}{|c|}{0} & \multicolumn{3}{|c|}{4} & \multicolumn{3}{|c|}{8} \\
\hline & Market order & Limit order & Total & Market order & Limit order & Total & Market order & Limit order & Total \\
\hline Slow traders & $4.085 \%$ & $95.915 \%$ & $100.00 \%$ & $86.608 \%$ & $13.392 \%$ & $100.00 \%$ & $88.254 \%$ & $11.746 \%$ & $100.00 \%$ \\
\hline Fast traders & $2.197 \%$ & $97.803 \%$ & $100.00 \%$ & $34.120 \%$ & $65.880 \%$ & $100.00 \%$ & $76.286 \%$ & $23.714 \%$ & $100.00 \%$ \\
\hline Total & $2.217 \%$ & $97.783 \%$ & $100.00 \%$ & $35.269 \%$ & $64.731 \%$ & $100.00 \%$ & $76.631 \%$ & $23.369 \%$ & $100.00 \%$ \\
\hline
\end{tabular}

\section{Table 4}

Changes in the type of order submission from traders due to HFT technology

The table presents the percentage of order types (market and limit orders) per trader in the scenarios with HFT (slow and fast traders in the market) and without HFT (only slow traders in the market). The Markov equilibrium and the posterior market simulation are obtained independently for each case.

\begin{tabular}{lccc}
\hline & \multicolumn{3}{c}{ \% of order types per trader characteristics } \\
\hline & \multicolumn{3}{c}{ Limit order } \\
\cline { 2 - 4 } & Market order & Slow traders and fast traders in the market \\
Slow traders & $47.583 \%$ & $52.417 \%$ & $100.00 \%$ \\
Fast traders & $17.145 \%$ & $82.855 \%$ & $100.00 \%$ \\
Total & $17.601 \%$ & $82.399 \%$ & $100.00 \%$ \\
& & Only slow traders in the market \\
Slow traders & $33.814 \%$ & $66.186 \%$ & $100.00 \%$ \\
\cline { 2 - 4 } & & &
\end{tabular}


Table 5

The effects of the speed components of HFT on the type of order submission per trader

The table presents the effects of the informational advantages and trading speed advantages of HFT traders on the percentage of order types (market and limit orders). The informational advantages of HFT traders are reflected in different lag periods $\Delta_{t}$ in which slow traders can observe the fundamental value of the asset, while fast traders know the contemporaneous value of $v_{t}$. The lag periods presented are $\Delta_{t}=0.0 \mathrm{sec}, \Delta_{t}=1.3 \mathrm{sec}$, and $\Delta_{t}=2.6 \mathrm{sec}$. The trading speed advantages of HFT traders are reflected in the different number of times that slow traders are 'slower' than fast traders in latency of submissions and modifications to previous limit orders. The difference in terms of trading speed advantages are performed by modifying the rate of the Poisson processes for the trader's arrivals $\left(\lambda^{H F T}\right.$ and $\lambda^{S T}$ for fast and slow traders, respectively) and the Poisson processes for the traders' re-enters $\left(\lambda_{r}^{H F T}\right.$ and $\lambda_{r}^{S T}$ for fast and slow traders, respectively). For instance, when slow and fast traders are equally fast in terms of trading speed $\lambda^{H F T}=\lambda^{S T}$ and $\lambda_{r}^{H F T}=\lambda_{r}^{S T}$, and when slow traders are 40 times 'slower' than traders $\lambda^{H F T}=40 \lambda^{S T}$ and $\lambda_{r}^{H F T}=40 \lambda_{r}^{S T}$. The Markov equilibrium and the posterior market simulation are obtained independently for each combination of parameters.

\begin{tabular}{|c|c|c|c|c|c|c|c|c|c|}
\hline & \multicolumn{9}{|c|}{ Slow traders and fast traders in the market } \\
\hline & Market order & Limit order & Total & Market order & Limit order & Total & Market order & Limit order & Total \\
\hline & \multicolumn{9}{|c|}{ Panel A:Information lag } \\
\hline & \multicolumn{3}{|c|}{$\Delta_{t}=0.0 \mathrm{sec}$} & \multicolumn{3}{|c|}{$\Delta_{t}=1.3 \mathrm{sec}$} & \multicolumn{3}{|c|}{$\Delta_{t}=2.6 \mathrm{sec}$} \\
\hline Slow traders & $39.322 \%$ & $60.678 \%$ & $100.00 \%$ & $47.583 \%$ & $52.417 \%$ & $100.00 \%$ & $48.088 \%$ & $51.912 \%$ & $100.00 \%$ \\
\hline Fast traders & $14.862 \%$ & $85.138 \%$ & $100.00 \%$ & $17.145 \%$ & $82.855 \%$ & $100.00 \%$ & $18.048 \%$ & $81.952 \%$ & $100.00 \%$ \\
\hline \multirow{3}{*}{ Total } & $15.079 \%$ & $84.921 \%$ & $100.00 \%$ & $17.601 \%$ & $82.399 \%$ & $100.00 \%$ & $18.505 \%$ & $81.495 \%$ & $100.00 \%$ \\
\hline & \multicolumn{9}{|c|}{$\begin{array}{l}\text { Panel B: Number of times that slow traders are slower than HFT traders in trading speed } \\
\text { (Low latency submissions and modifications of previous limit orders) }\end{array}$} \\
\hline & \multicolumn{3}{|c|}{1} & \multicolumn{3}{|c|}{20} & \multicolumn{3}{|c|}{40} \\
\hline Slow traders & $22.273 \%$ & $77.727 \%$ & $100.00 \%$ & $47.583 \%$ & $52.417 \%$ & $100.00 \%$ & $48.924 \%$ & $51.076 \%$ & $100.00 \%$ \\
\hline Fast traders & $15.554 \%$ & $84.446 \%$ & $100.00 \%$ & $17.145 \%$ & $82.855 \%$ & $100.00 \%$ & $18.636 \%$ & $81.364 \%$ & $100.00 \%$ \\
\hline Total & $18.448 \%$ & $81.552 \%$ & $100.00 \%$ & $17.601 \%$ & $82.399 \%$ & $100.00 \%$ & $18.916 \%$ & $81.084 \%$ & $100.00 \%$ \\
\hline
\end{tabular}

Table 6

Changes in the bid-ask spread and the limit order book depth due to HFT technology

The table reports the bid-ask spread and the number of limit orders at the ask price in the scenarios with HFT (slow and fast traders in the market) and without HFT (only slow traders in the market). The bid-ask spread is the average of the difference between the bid and the ask quotes in the book over time which is measured in ticks. This table also presents the average number of limit orders at the ask quote. The market is observed every 10 minutes. The table only shows the number of limit orders at the ask since the model is symmetric, and thus the data from the buy and sell sides are similar. The Markov equilibrium and the posterior market simulation are obtained independently for each case.

\begin{tabular}{llc} 
& & \multicolumn{2}{c}{ Bid-ask spread } & N. of limit orders at the ask \\
\cline { 2 - 3 } Slow and Fast traders & \multicolumn{2}{c}{ Slow traders and fast traders in the market } \\
\cline { 2 - 3 } Slow traders & 1.326 & 4.121 \\
\cline { 2 - 3 } & \multicolumn{3}{c}{ Only slow traders in the market } \\
\hline
\end{tabular}


Table 7

The effects of the speed components of HFT on the bid-ask spread and the limit order book depth

The table presents the effects of the informational advantages and trading speed advantages of HFT traders on the bid-ask spread and the number of limit orders at the ask price. The bid-ask spread is the average of the difference between the bid and the ask quotes in the book over time which is measures in ticks. This table also presents the average number of limit orders at the ask quote. The market is observed every 10 minutes. The table only shows the number of limit orders at the ask since the model is symmetric, and thus the data from the buy and sell sides are similar. The informational advantages of HFT traders are reflected in different lag periods $\Delta_{t}$ in which slow traders can observe the fundamental value of the asset, while fast traders know the contemporaneous value of $v_{t}$. The lag periods presented are $\Delta_{t}=0.0 \mathrm{sec}, \Delta_{t}=1.3 \mathrm{sec}$, and $\Delta_{t}=2.6 \mathrm{sec}$. The trading speed advantages of HFT traders are reflected in the different number of times that slow traders are 'slower' than fast traders in latency of submissions and modifications to previous limit orders. The difference in terms of trading speed advantages are performed by modifying the rate of the Poisson processes for the traders' arrivals $\left(\lambda^{H F T}\right.$ and $\lambda^{S T}$ for fast and slow traders, respectively) and the Poisson processes for the traders' re-enters $\left(\lambda_{r}^{H F T}\right.$ and $\lambda_{r}^{S T}$ for fast and slow traders, respectively). For instance, when slow and fast traders are equally fast in terms of trading speed $\lambda^{H F T}=\lambda^{S T}$ and $\lambda_{r}^{H F T}=\lambda_{r}^{S T}$, and when slow traders are 40 times 'slower' than traders $\lambda^{H F T}=40 \lambda^{S T}$ and $\lambda_{r}^{H F T}=40 \lambda_{r}^{S T}$. The Markov equilibrium and the posterior market simulation are obtained independently for each combination of parameters.

\begin{tabular}{|c|c|c|c|}
\hline & \multicolumn{3}{|c|}{ Slow traders and fast traders in the market } \\
\hline & \multicolumn{3}{|c|}{ Panel A:Information lag } \\
\hline & $\Delta_{t}=0.0 \mathrm{sec}$ & $\Delta_{t}=1.3 \mathrm{sec}$ & $\Delta_{t}=2.6 \mathrm{sec}$ \\
\hline Bid-ask spread & 1.292 & 1.326 & 1.354 \\
\hline \multirow[t]{3}{*}{ N. of limit orders at the ask } & 4.910 & 4.121 & 3.838 \\
\hline & \multicolumn{3}{|c|}{$\begin{array}{l}\text { Panel B: Number of times that slow traders are slower than HFT traders in trading speed (Low } \\
\text { latency submissions and modifications of previous limit orders) }\end{array}$} \\
\hline & 1 & 20 & 40 \\
\hline Bid-ask spread & 1.380 & 1.326 & 1.303 \\
\hline N. of limit orders at the ask & 3.326 & 4.121 & 4.155 \\
\hline
\end{tabular}

Table 8

Average payoff for different traders in a market with and without HFT

The table contains average payoff in ticks for diverse types of traders differenced by private values, $\alpha$, in the scenarios with HFT (slow and fast traders in the market) and without HFT (only slow traders in the market). We combine the results for positive and negative private values given that the model is symmetric on both sides of the book. The Markov equilibrium and the posterior market simulation are obtained independently for each case. Standard errors are less than 0.0007 for fast traders, while standard errors for slow traders are less than $0.0024(0.0032)$ in the scenario of slow and fast traders in the market (only slow traders in the market).

\begin{tabular}{|c|c|c|c|c|}
\hline & \multicolumn{3}{|c|}{ Private value $|\alpha|$} & \multirow[b]{2}{*}{ Total } \\
\hline & 0 & 4 & 8 & \\
\hline & \multicolumn{4}{|c|}{ Slow traders and fast traders in the market } \\
\hline Slow traders & 0.240 & 3.366 & 7.301 & 3.614 \\
\hline Fast traders & 0.370 & 3.589 & 7.537 & 3.809 \\
\hline \multirow[t]{2}{*}{ Total } & 0.366 & 3.582 & 7.529 & 3.803 \\
\hline & \multicolumn{4}{|c|}{ Only slow traders in the market } \\
\hline Slow traders & 0.983 & 2.311 & 4.929 & 2.698 \\
\hline
\end{tabular}


Table 9

Average payoff for different traders in a market in the absence of slow traders' capacity to improve the accuracy of their beliefs through learning

The table contains average payoff in ticks for diverse types of traders when slow traders have no capacity to improve the accuracy of their beliefs through learning. Traders are differenced by private values. The table presents the results in the scenarios with HFT (slow and fast traders in the market) and without HFT (only slow traders in the market). We combine the results for positive and negative private values given that the model is symmetric on both sides of the book. The Markov equilibrium and the posterior market simulation are obtained independently for each case. Standard errors are less than 0.0007 for fast traders, while standard errors for slow traders are less than $0.0024(0.0032)$ in the scenario of slow and fast traders in the market (only slow traders in the market).

\begin{tabular}{|c|c|c|c|c|}
\hline & \multicolumn{3}{|c|}{ Private value $|\alpha|$} & \multirow[b]{2}{*}{ Total } \\
\hline & 0 & 4 & 8 & \\
\hline & \multicolumn{4}{|c|}{ Slow traders and fast traders in the market } \\
\hline & \multicolumn{4}{|c|}{ Slow traders cannot learn from the trading activity of HFT to improve the accuracy about $v_{t}$} \\
\hline Slow traders & -0.191 & 3.214 & 7.279 & 3.408 \\
\hline Fast traders & 0.328 & 3.598 & 7.580 & 3.813 \\
\hline \multirow[t]{2}{*}{ Total } & 0.314 & 3.587 & 7.572 & 3.802 \\
\hline & \multicolumn{4}{|c|}{ Only slow traders in the market } \\
\hline Slow traders & 0.983 & 2.311 & 4.929 & 2.698 \\
\hline
\end{tabular}

\section{Table 10}

The impact of the speed components of HFT on the average payoff with and without slow traders' capacity to improve the accuracy of their beliefs through learning

The table shows the impact of the informational advantages and trading speed advantages of HFT traders on the average payoff, in the case with (Panel A) and without (Panel B) slow traders' capacity to improve the accuracy of their beliefs through learning. The average payoff is expressed in ticks. The informational advantages of HFT traders are reflected in different lag periods $\Delta_{t}$ in which slow traders can observe the fundamental value of the asset, while fast traders know the contemporaneous value of $v_{t}$. The trading speed advantages of HFT traders are reflected in the different number of times that slow traders are 'slower' than fast traders in latency of submissions and modifications to previous limit orders. The difference in terms of trading speed advantages are performed by modifying the rate of the Poisson processes for the traders' arrivals ( $\lambda^{H F T}$ and $\lambda^{S T}$ for fast and slow traders, respectively) and the Poisson processes for the traders' re-enters $\left(\lambda_{r}^{H F T}\right.$ and $\lambda_{r}^{S T}$ for fast and slow traders, respectively). For instance, when slow and fast traders are equally fast in terms of trading speed $\lambda^{H F T}=\lambda^{S T}$ and $\lambda_{r}^{H F T}=\lambda_{r}^{S T}$, and when slow traders are 40 times 'slower' than traders $\lambda^{H F T}=40 \lambda^{S T}$ and $\lambda_{r}^{H F T}=40 \lambda_{r}^{S T}$. The Markov equilibrium and the posterior market simulation are obtained independently for each combination of parameters. Standard errors are less than 0.0009 for fast traders, while standard errors for slow traders are less than 0.0037 .

\begin{tabular}{|c|c|c|c|}
\hline & \multicolumn{3}{|c|}{ Slow traders and fast traders in the market } \\
\hline & \multicolumn{3}{|c|}{ Total payoff } \\
\hline & \multirow{3}{*}{ Base case } & & Slow traders are equally fast as \\
\hline & & No informational advantages & HFT Traders \\
\hline & & $\Delta_{t}=0.0 \mathrm{sec}$ & $\begin{array}{l}\text { in latency submissions } \\
\text { and modifications of orders }\end{array}$ \\
\hline & \multicolumn{3}{|c|}{ Panel A: Slow traders can learn from the trading activity of HFT to improve the accuracy about $v_{t}$} \\
\hline Fast traders & 3.809 & 3.798 & 3.891 \\
\hline \multirow[t]{2}{*}{ Total } & 3.803 & 3.795 & 3.889 \\
\hline & \multicolumn{3}{|c|}{ Panel B: Slow traders cannot learn from the trading activity of HFT to improve the accuracy about $v$} \\
\hline Slow traders & 3.408 & 3.636 & 3.752 \\
\hline Fast traders & 3.813 & 3.798 & 3.967 \\
\hline Total & 3.802 & 3.795 & 3.859 \\
\hline
\end{tabular}




\section{Table 11}

The impact of different cancellation fees on the percentage of cancellations per trader The table contains the percentage of cancellations per number of traders. Cancellation fees are measured in ticks. The Markov equilibrium and the posterior market simulation are obtained in the scenario when there slow and fast traders in the market.

\begin{tabular}{lccc}
\hline & \multicolumn{3}{c}{ Slow traders and fast traders in the market } \\
\cline { 2 - 4 } & \% of Cancellations per N. of Traders \\
\cline { 2 - 4 } Slow traders & Cancellation fee=0.0 & Cancellation fee=0.1 & Cancellation fee=0.2 \\
Fast traders & $35.493 \%$ & $23.191 \%$ & $19.752 \%$ \\
Total & $73.973 \%$ & $56.731 \%$ & $46.134 \%$ \\
\hline
\end{tabular}

Table 12

\section{Changes in market quality measures due to different cancellation fees}

The table presents changes due to different cancellation fees on microstructure noise $\left(p_{t}-v_{t}\right)$, slow traders' errors in beliefs $\left(\mathrm{E}\left(v_{t}\right)-v_{t}\right)$, the percentage of order types (market and limit orders) the bidask spread, the number of limit orders at the ask price, payoffs, and the percentage of cancellations per trader. Cancellation fees are measured in ticks. The variables $p_{t}, v_{t}$, are $\mathrm{E}\left(v_{t}\right)$ are defined in Table 1 and expressed in ticks. The market is observed every 10 minutes to obtain microstructure noise, slow traders' errors in beliefs, the bid-ask spread, and the number of limit orders at the ask price. The Markov equilibrium and the posterior market simulation are obtained independently for each combination of parameters.

\begin{tabular}{lccc}
\hline & \multicolumn{3}{c}{ Slow traders and fast traders in the market } \\
\hline & \multicolumn{3}{c}{ Cancellation fee } \\
\cline { 2 - 3 } Mean $\left|v_{t}-p_{t}\right|$ & Cancellation fee=0.0 & Cancellation fee=0.1 & Cancellation fee=0.2 \\
\cline { 2 - 3 } Std. Dev. $\left(v_{t}-p_{t}\right)$ & 0.492 & 0.533 & 0.580 \\
Mean $\left|\mathrm{E}\left(v_{t}\right)-v_{t}\right|$ & 0.712 & 0.747 & 0.785 \\
Std. Dev $\left(\mathrm{E}\left(v_{t}\right)-v_{t}\right)$ & 0.190 & 0.224 & 0.252 \\
N. of market orders / N. of orders (\%) & 0.355 & 0.387 & 0.413 \\
N. of limit orders / N. of orders (\%) & $14.937 \%$ & $17.601 \%$ & $20.518 \%$ \\
Bid-ask spread & $85.063 \%$ & $83.399 \%$ & $79.482 \%$ \\
N. of limit orders at the ask & 1.298 & 1.326 & 1.382 \\
Payoff (slow traders) & 4.595 & 4.121 & 3.381 \\
Payoff (fast traders) & 3.608 & 3.614 & 3.618 \\
Payoff (total) & 3.811 & 3.809 & 3.791 \\
\end{tabular}


Table 13

Market quality measures when there are different unexpected volatility shocks in the fundamental value of asset

The table reports the impact of different unexpected volatility shocks in the fundamental value of the asset, $\Delta \sigma$, on microstructure noise $\left(p_{t}-v_{t}\right)$, slow traders' errors in beliefs $\left(\mathrm{E}\left(v_{t}\right)-v_{t}\right)$, the percentage of order types (market and limit orders) the bid-ask spread, the number of limit orders at the ask price, payoffs, and the percentage of cancellations per trader. The variables $p_{t}, v_{t}$, are $\mathrm{E}\left(v_{t}\right)$ are defined in Table 1 and expressed in ticks. The market is observed every 10 minutes to obtain microstructure noise, slow traders' errors in beliefs, the bid-ask spread, and the number of limit orders at the ask price. The volatility shock is performed as follows. The Markov equilibrium for the trading game is calculated when there are slow and fast traders in the market, with a level of volatility in the fundamental value of the asset, $\sigma$, equal to 0.50 . However, the posterior simulations using the model equilibrium are performed using superior levels of volatility $(\sigma+\Delta \sigma)$. Therefore, traders do not expect this higher volatility in the market. This unexpected higher volatility represents our volatility shock. The base case is presented in the second column. The third and fourth columns report the effect on multiple quality measures of a shock equal to $0.10(\sigma+\Delta \sigma=0.60)$ and a shock equal to $0.20(\sigma+\Delta \sigma=0.70)$, respectively.

\begin{tabular}{|c|c|c|c|}
\hline & \multicolumn{3}{|c|}{ Slow traders and fast traders in the market } \\
\hline & \multicolumn{3}{|c|}{ Shock in the Volatility of the Fundamental Value $(\sigma+\Delta \sigma)$} \\
\hline & 0.50 & 0.60 & 0.70 \\
\hline Mean $\left|v_{t}-p_{t}\right|$ & 0.533 & 0.556 & 0.580 \\
\hline Std. Dev. $\left(v_{t}-p_{t}\right)$ & 0.747 & 0.772 & 0.799 \\
\hline Mean $\left|\mathrm{E}\left(v_{t}\right)-v_{t}\right|$ & 0.224 & 0.293 & 0.350 \\
\hline Std. Dev $\left(\mathrm{E}\left(v_{t}\right)-v_{t}\right)$ & 0.387 & 0.460 & 0.519 \\
\hline N. of market orders / N. of orders (\%) & $17.601 \%$ & $21.066 \%$ & $24.117 \%$ \\
\hline N. of limit orders / N. of orders (\%) & $83.399 \%$ & $78.934 \%$ & $75.883 \%$ \\
\hline Bid-ask spread & 1.326 & 1.367 & 1.424 \\
\hline N. of limit orders at the ask & 4.121 & 3.148 & 2.572 \\
\hline Payoff (slow traders) & 3.614 & 3.609 & 3.606 \\
\hline Payoff (fast traders) & 3.809 & 3.834 & 3.853 \\
\hline Payoff (total) & 3.803 & 3.826 & 3.845 \\
\hline N. of cancelat. / N. of traders (slow traders) & $23.191 \%$ & $23.364 \%$ & $23.597 \%$ \\
\hline N. of cancelat. / N. of traders (fast traders) & $56.731 \%$ & $51.683 \%$ & $49.699 \%$ \\
\hline N. of cancelat. / N. of traders (total) & $55.703 \%$ & $50.759 \%$ & $48.812 \%$ \\
\hline
\end{tabular}

